\title{
SCIENTIFIC REPORTS

\section{OPEN Interactions of a medicinal climber Tinospora cordifolia with supportive interspecific plants trigger the modulation in its secondary metabolic profiles}

\begin{abstract}
Bhawana Sharma, Aarti Yadav \& Rajesh DaburiD
Tinospora cordifolia (TC) is scientifically proven immunomodulatory drug being used for centuries. Ancient literature reported that inter-specific interactions change medicinal properties of TC. Thus, the current study is aimed to understand the influence of interspecific biotic interactions on chemo-profiles of TC. To explore it, TC samples collected from six co-occurring plants, i.e. Azarditchita indica, Acacia nilotica, Albezia lebbeck, Ficus benghalensis, Tamarandus indica and Acacia leucophloea were analyzed by HPLC-ESI-OTOF-MS. Mass data were subjected to multivariate analysis. Support vector machines (SVMs) was found to be best classifier $\left(r^{2}<0.93\right)$. Data analysis showed the specific compounds in all $T C$ due to inter-specific interactions. Data were further analyzed with SNK post-hoc test followed by permutative $(n=50)$ Bonferroni FDR multiple testing correction. The compound without any missing values reduced the number of variables to $133(p<0.01)$. Statistical analysis revealed that $T C$ having interactions with A.lebbeck and A. nilotica formed the most distant groups. However, TC co-occurred with $A$. indica showed the highest number of up-regulated metabolites, including jatrorrhizine, chrysin, peonidin, 6-methylcoumarin and some terpenoids. Some metabolites, including jatrorrhizine and magnoflorine were quantified to confirm the accuracy of qualitative analysis. Results demonstrated the influence of inter-specific biotic interactions on TC chemo-profiles, hence its medicinal properties.
\end{abstract}

Tinospora cordifolia (Willd.) Miers, a climbing shrub of the family Menispermaceae, is a well known Ayurvedic medicinal herb with different names including rasayana (to purify the blood), amrita (to bring the dead back to life), nectar of immortality and heavenly elixir. Ayurveda and Chinese traditional medicinal systems describe the use of T. cordifolia not only as a health tonic but also for the treatment of a large number of diseases including diabetes, asthma, liver and platelet damages, stress and cancer ${ }^{1}$. The plant has been reported for its diverse pharmacological properties, i.e. immunomodulatory, hepatoprotective, neuroprotective and nephroprotective ${ }^{2-6}$. T. cordifolia that grows in co-occurrence with Azaditchia. indica and Magnifera indica is the best for medicinal efficacy in Ayurveda. Recently, it has been shown that T. cordifolia showed best immunomodulatory activity on interaction with $A$. indica $a^{7,8}$. Our lab has documents that phytochemical constituents (i.e. tinosporaside) were significantly higher when $T$. cordifolia co-occured with $A$. indica $a^{9}$. These studies indicate that the medicinal properties of $T$. cordifolia are affected by interspecific interactions with other plants. Till date no work has been undertaken to explore the the variations in phytochemicals of T. cordifolia due to inter-specific interactions with higher plants.

The available biomarker for quality control of T. cordifolia doesn't seem to be sufficient and reliable due to variations in chemicals as result of its geographical location, climate and biotic interactions with higher plants. These issues have not been addressed during the slection of biomarkers. Therefore, to eplore the chemo-profiles of T. cordifolia and to identify reliable biomarkers, a highly sophisticated tool, high performance liquid chromatography coupled with quadrupole time of flight mass spectrometer (HPLC-ESI-QTOF-MS) has been used. This tool not only provides high mass accuracy and resolution of mass fragments but can yield empirical chemical 
formulae to facilitate the structural elucidation even without the use of reference standards ${ }^{10}$. Furthermore, statistical analysis of mass data can recognize important markers of plants grown in different conditions ${ }^{11}$. Thus, the current study was aimed to sepeficlly evaluate the alterations in the secondary metabolites of T. cordifolia co-occurred with other plants. In order to understand the comprehensive impact of biotic interactions on the chemo-profiles of T. cordifolia, the samples were collected and analyzed using HPLC-ESI-QTOF-MS. The data was analyzed using principal component analysis (PCA) and multivariate analysis followed by SNK post-hoc test with permutative $(n=50)$ Bonferroni FDR multiple testing correction to compare chemoprofiles among the groups. Hence, study was focused to explore the changes in the chemoprofiles due to interspecific interactions of T. cordifolia co-occurred with other higher plants.

\section{Results}

General characteristics of TCEs and HPLC method development. Freshly prepared TCE was brown in color, $\mathrm{pH} 7.57$, slightly bitter in taste and without any characteristic odour. Its specific gravity and viscosity were recorded to be 1.2 and $1.6 \mathrm{cP}$. Our previous studies demonstrated that most of metabolites of T. cordifolia have been detected in positive ion polarity mode due to greater sensitivity to the signals as compared with the negative ion ${ }^{5,12}$. Therefore, total ion current chromatograms (TIC) of all the groups, i.e. control, AIN, ALL, ALC, ANI, TMI, and FBG were acquired in positive ion polarity mode. Water and acetonitrile with $0.1 \%$ formic acid selected as mobile phase as these solvents provided low background noise and better chromatographic peaks. Visual examination of base peaks of chromatograms extracted from TIC showed metabolite variations among the groups (Fig. 1). Intra and inter-day precision and accuracy were calculated by injecting a mixture of standards three times in a day for three consecutive days. Intra and inter-day precision was within 0.31 and $0.85 \%$, while accuracies were more than 97.5 to $100 \%$. Relative standard deviation (RSD) of repeatability of five different solutions was less than $1.57 \%$. Recovery of the method was established by adding three different concentrations of reference standards to the crude extracts of T. cordifolia. The recovery of standards was found in between 98.07 to $100.07 \%$, with RSD less than $2.63 \%$ (Table 1 ).

Multivariate statistical analysis. Fifty nine major peaks were observed in the chromatogram when integrated. All the spectra were aligned using ion intensity, retention time $(<0.2 \mathrm{~min})$ and mass $(<5 \mathrm{ppm})$ with the help of internal standards i.e. ions of $m / z 296.15,373.13,311.13,230.24$, and 436.44 present universally in all the samples. Final data were normalized using Z-transforms. Data sets were subjected to one way ANOVA $(p<0.05)$, fold change $(>2.0)$ and coefficient variation $(>15 \%)$ analysis. ALL and ANI groups showed the highest number of down-regulated metabolites. Box Whisker plots of the data revealed least variability in the ALL group as compared to other samples. All the groups showed more variability in the upper quartile portion of Box Whisker plot (Fig. 2A). Supervised PCA was performed on all the datasets and visualized to check for outliers and classification trend among the samples (Table S1). Principal components have been extracted from the variables in the datasets. Statistical analysis involves principal component analysis projection to latent structures for identifying variation in spectral features of samples. PCA of 7 groups resulted in 1643 principal components. Each groups was observed to be distinct, forming their own cluster and lying far apart from each other. All the groups showed $23.42,17.19$ and $13.62 \%$ variations along the $\mathrm{X}, \mathrm{Y}$ and $\mathrm{Z}$ axis respectively (Fig. 2B). Supervised PCA plot showed highest variations in AIN and ALC groups as compared to other groups. Further, data were subjected to multivariate analysis to identify and reveal differentially expressed metabolites in different groups. Initial analysis showed confidence in variability among the groups and the presence of distinct metabolites. Already established separation among the groups was sharpened by multivariate analysis. Data was further subjected to PLS-DA, SVM, NB, DT and NN classifiers for preparing respective classifier models ${ }^{13}$. Classifier models expressed confidence ranging from 1 to 0.896 (Table S2). All the models were trained for further prediction of unknown samples. Mass data files of different plant extracts and T. cordifolia (collected from different supporting trees) extracts were subjected to the trained models for classification and identification. A trained model has classified all the unknown samples and prediction measure were expressed as confidence measure. Trained model of PLS-DA, NB and DT failed to classify all known samples (Table S3), whereas SVM and NB classified all the extracts correctly and showed least $\mathrm{r}^{2}$ for the samples other than T. cordifolia (Table S2). Furthermore, SVM was found to be a better discrimination model and powerful classification tool in real-world applications due to expression of better confidence levels in T. cordifolia samples and least confidence in other extracts along with its excellent learning performance as reported earlier ${ }^{14}$. SVM data shown to have 1083 ranked metabolites from all the seven samples. Being best model, SVM data were subjected to further analysis to reduce the number of metabolites and to keep only significant variables.

Differential metabolites of $T$. cordifolia due biotic interactions with different trees. Venn diagrammatic representation of specific metabolites in respective groups showed the presence of 168, 167, 59, $126,83,80$, and 150 differentially expressed metabolites in CON, AIN, ALL, ALC, ANI, TMI and FBG groups respectively. It showed the metabolites present in specific groups only, and/or may be due to missing values in data. Venn diagrammatic representation showed the presence of $6,2,7,5,5,3$, and 0 metabolites exclusively in CON, AIN, ALL, ALC, ANI, TMI and FBG groups, respectively (Fig. 3). It showed that 8-hydroxytinosporide and one unknown terpenoid were exclusively present in the AIN group while the control group contains 5-allyloxysalvigenin, trans-farnesol, reticuline, $\mathrm{N}$-isovaleroylglycine and two unknown metabolites. The only ANI group contained palmatoside $\mathrm{C}, \alpha$-D-glucan, 5-aminovaleric acid and two unknown metabolites of higher molecular weight while, ALL group contained tinosporinone, baenzigeroside A, tinosinen, tinoridine and two unknown metabolites. The TMI group contains 11-hydroxymustakone along with two unknown metabolites (Table 2). Data were subjected to One-way ANOVA with SNK post-hoc test and asymptotic $p$ values were computed with permutative $(n=50)$ Bonferroni FDR multiple testing correction. After analysis, 229 metabolites 


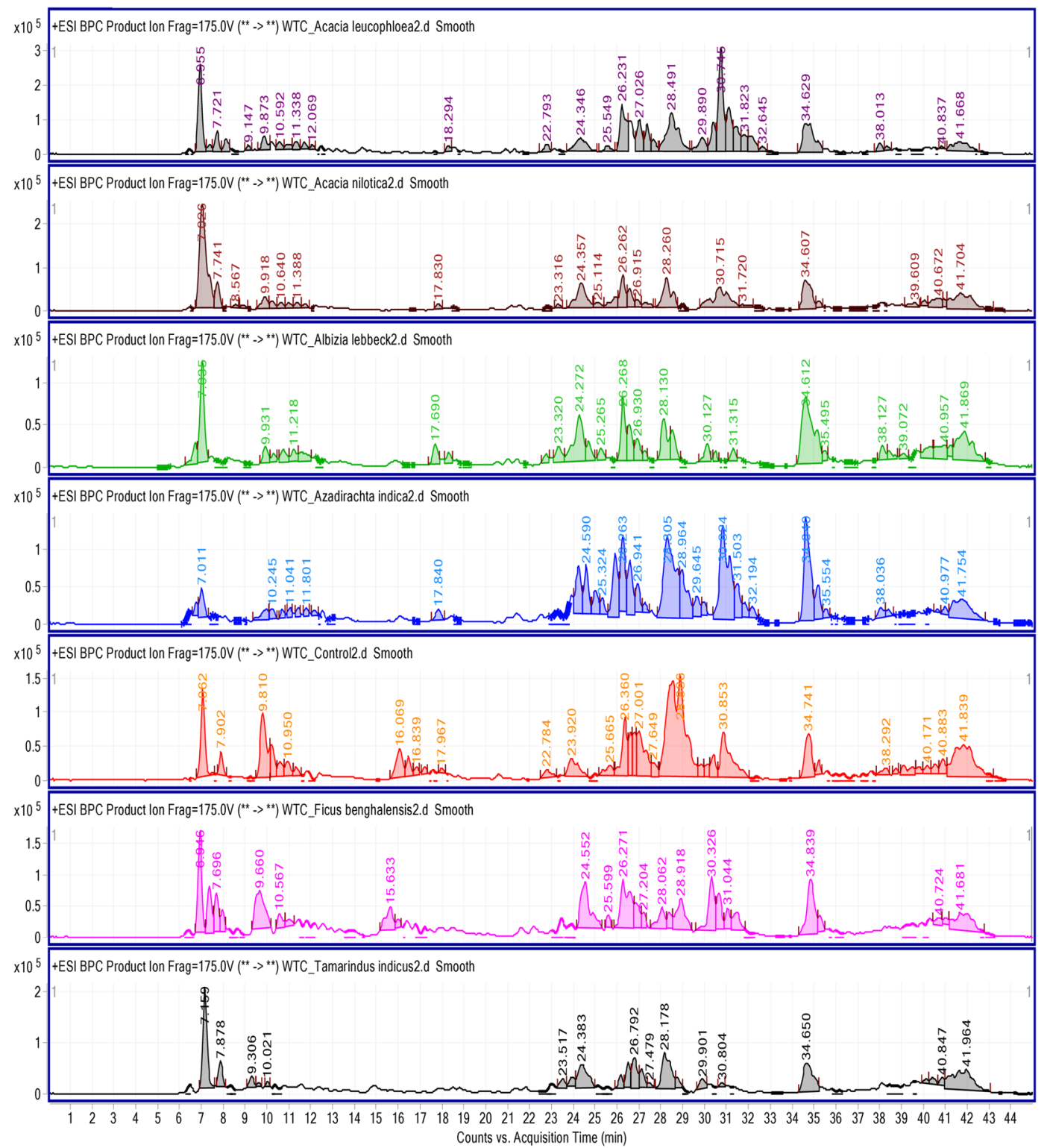

Figure 1. Base peak chromatograms of all seven groups extracted from total ion current chromatograms (TIC), showing visual differences among the chromatographic profiles of various groups. A, B, C, D, E, F and G represents the ALC, ANI, ALL, AIN, CON, FBG, TMI groups respectively.

\begin{tabular}{|c|c|c|c|c|c|c|c|c|}
\hline \multirow[b]{2}{*}{ S. No. } & \multirow[b]{2}{*}{ Compounds } & \multicolumn{2}{|c|}{ Intra-day $(\mathrm{n}=9)$} & \multicolumn{2}{|c|}{ Inter-day $(\mathbf{n}=9)$} & \multirow{2}{*}{\begin{tabular}{|l}
$\begin{array}{l}\text { Repeatability } \\
(\mathbf{n}=9)\end{array}$ \\
RSD\% \\
\end{tabular}} & \multicolumn{2}{|l|}{ Recovery } \\
\hline & & \begin{tabular}{|l|}
$\begin{array}{l}\text { Accuracy } \\
\text { (RE\%) }\end{array}$ \\
\end{tabular} & \begin{tabular}{|l|}
$\begin{array}{l}\text { Precision } \\
\text { (RSD\%) }\end{array}$ \\
\end{tabular} & \begin{tabular}{|l|}
$\begin{array}{l}\text { Accuracy } \\
\text { (RE\%) }\end{array}$ \\
\end{tabular} & $\begin{array}{l}\begin{array}{l}\text { Precision } \\
\text { (RSD\%) }\end{array} \\
\end{array}$ & & \begin{tabular}{|l|} 
Mean \\
Recovery (\%)
\end{tabular} & RSD\% \\
\hline 1. & Berberine & 99.1 & 0.22 & 99.4 & 0.46 & 0.61 & 99.63 & 1.53 \\
\hline 2. & Palmatine & 97.4 & 0.17 & 100.4 & 0.43 & 1.57 & 99.31 & 1.46 \\
\hline 3. & Jatrorrhizine & 99.5 & 0.11 & 100.5 & 0.53 & 1.27 & 100.07 & 1.87 \\
\hline 4. & Magnoflorine & 98.7 & 0.16 & 99.7 & 0.85 & 0.95 & 99.21 & 2.63 \\
\hline 5. & Choline & 96.5 & 0.31 & 97.5 & 0.67 & 0.83 & 98.07 & 1.62 \\
\hline
\end{tabular}

Table 1. Statistical results of inter and intraday precision, accuracy and recovery of five important compounds of T. cordifolia.

with $\mathrm{p}<0.001$ and fold change $>2.0$ were found to be differentially regulated. Missing values may originate from analytical, computational and biological backgrounds, therefore, cause problem in mass spectrometry data analysis. Mean imputation is the substitute of missing values. However, in the current study, entities with missing 

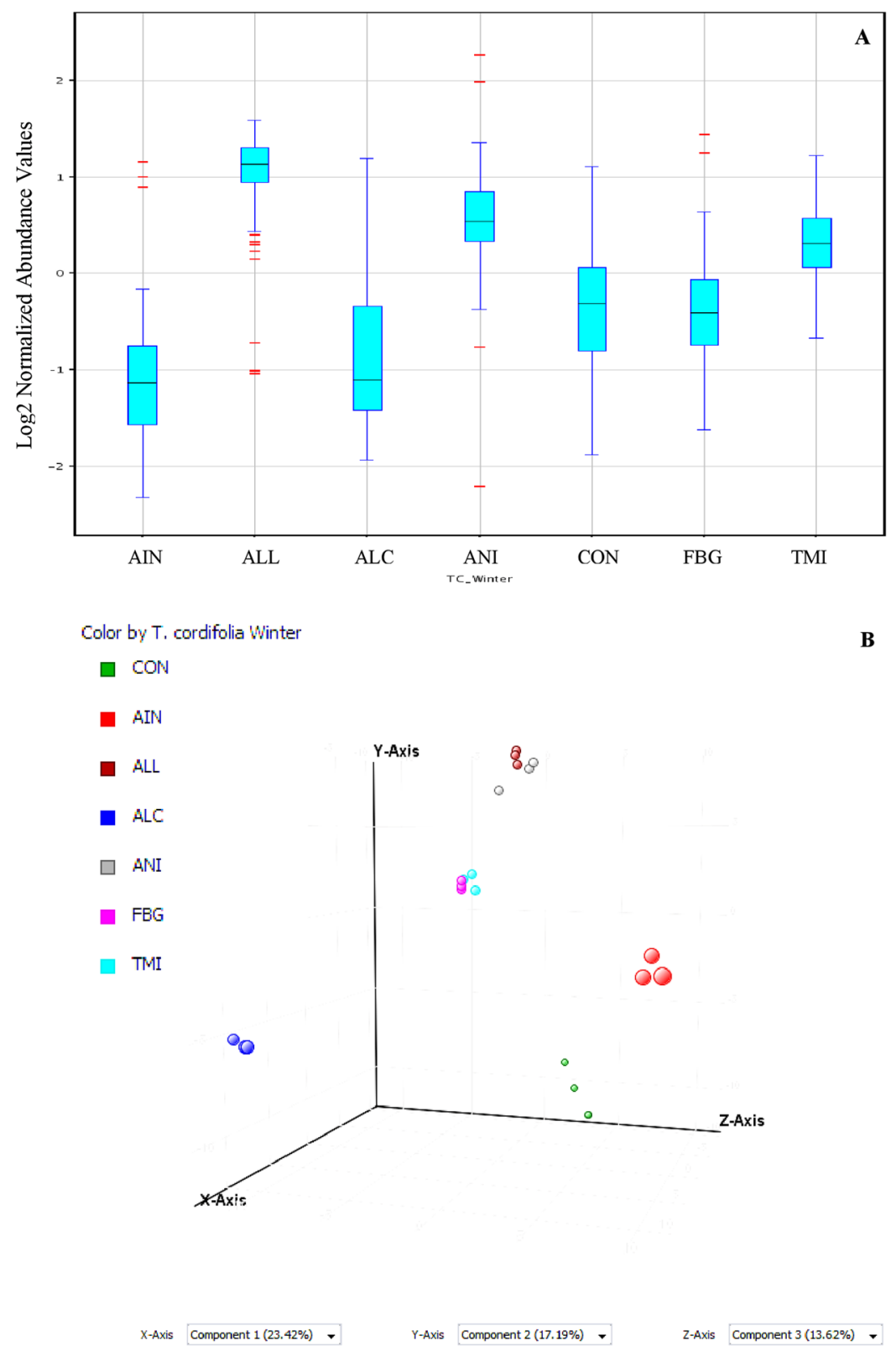

Figure 2. (A) Box Whisker plots of various groups after filtration, significance and fold change analysis showing high variations across the groups. (B) Principle component analysis score plot representing \% variations among the metabolites of all seven groups where variability along the $\mathrm{x}, \mathrm{y}$ and $\mathrm{z}$ axis are $23.42 \%$, $17.19 \%$ and $13.62 \%$ respectively. AIN, ALC, ALL and ANI represents the distant groups showing highest variations.

values were removed due to the high number of variables that reduced the number of discriminated metabolite to 133 (Table 3). The FBG group did not contain any specific metabolite. Observed mass differences and fragment ions are given in Table S3. Spearman correlation heat map of groups and metabolites without missing values is shown in Fig. 4. Spearman correlation heatmap analysis showed that TMI and FBG, control and ALC groups were close to each other, whereas ALL and ANI being more distant groups. Clusters of metabolites in red color and inter-spreading metabolites in blue color across the different groups are highly variable regions showing differential expression of metabolites in that particular group (Fig. 4). Differential metabolites without missing values were identified with the help of authentic standards and different standard databases [METLIN and MassBank] (https://metlin.scripps.edu/; http://www.massbank.jp/) ${ }^{15,16}$ by analyzing molecular formula, isotopic and fragmentation pattern (Table S4).

Differential metabolites in AIN group. Along with 8-hydroxytinosporide and one unknown terpenoid present exclusively in this group. Protonated molecule $[\mathrm{M}+\mathrm{H}]^{+}$at $m / z 391.164$ was identified as 8-hydroxytinosporide due the presence of characteristic mass ions at $m / z 373\left[\mathrm{M}-\mathrm{H}_{2} \mathrm{O}\right]^{+}, 355\left[\mathrm{M}-\mathrm{H}_{2} \mathrm{O}\right]^{+}, 347$ 


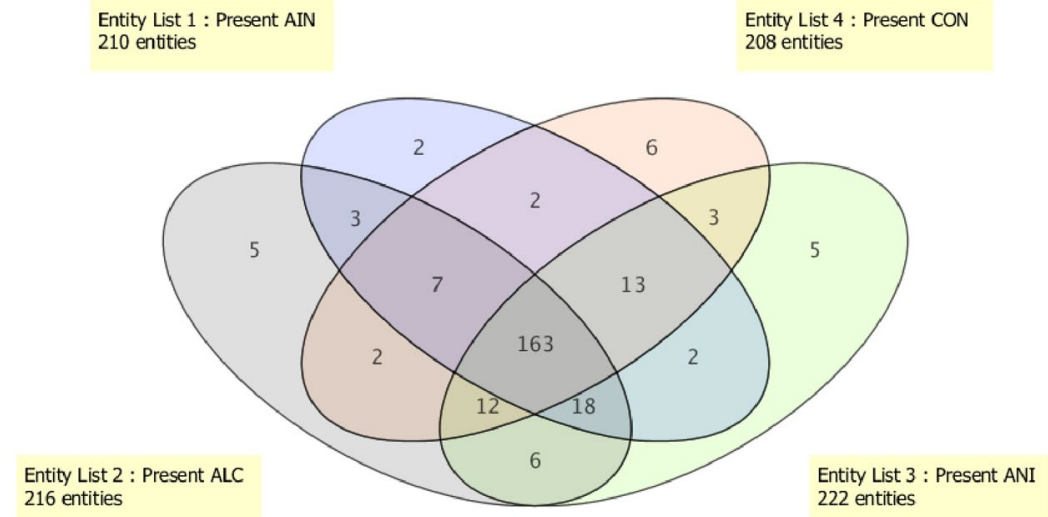

Entity List 1 : Present CON

Entity List 4 : Present ALL 208 entities 209 entities

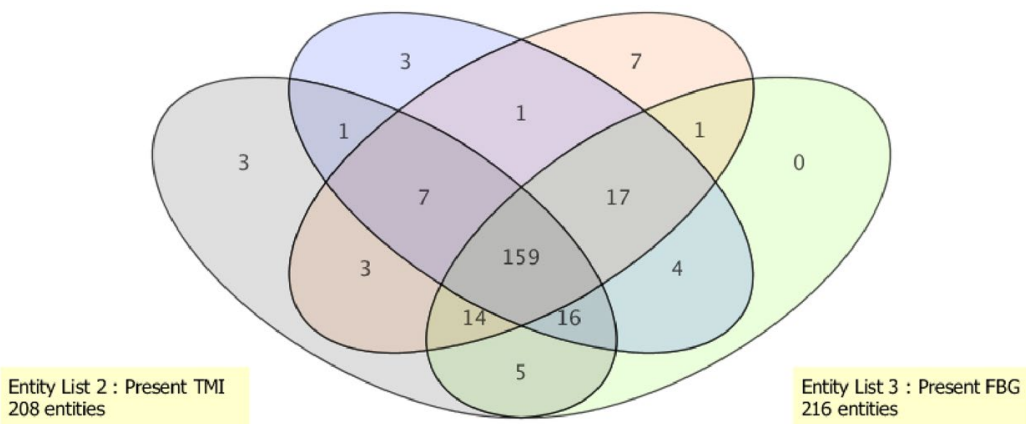

Figure 3. Venn diagram showing distribution of number of specific entities among seven different groups. The diagram shows overlapping and distinct metabolites indicated by the numbers in the intersections and circles, respectively.

$\left[\mathrm{M}-\mathrm{CO}_{2}\right]^{+}, 253$ and 123 as reported earlier ${ }^{17}$. Statistical analysis showed presence of 18 compounds up-regulated by $>2.0$ fold in AIN group. High levels ( $>3$ fold) of borapetosides D, N-methylcoclaurine, isotanshinone IIA, peonidin and 5-allyloxysalvigenin derivative were found in this group (Tables 3 and S2). Borapetoside D is a clerodane-type furanoditerpene containing two D-glucopyranose units, hence consecutive loss of $162 \mathrm{Da}$ from parent ion $[\mathrm{M}+\mathrm{H}]^{+}$of $m / z 698$ generate positive ion fragments at $m / z 375$ that further generate ions at $\mathrm{m} / z 357$ $\left[\mathrm{M}-\mathrm{Glu}-\mathrm{Glu}-\mathrm{H}_{2} \mathrm{O}\right]^{+}, 343\left(\mathrm{CH}_{4}\right), 265,206,185,159,149,121$ and 105 as reported earlier ${ }^{18}$. N-Methylcoclaurine resolved at $24.4 \pm 0.2 \mathrm{~min}$ in all the groups and parent ion $[\mathrm{M}+\mathrm{H}]^{+}$at $\mathrm{m} / z 299.16$ further produced positive ions at $m / z 282,271,257$ due to loss of $\mathrm{H}_{2} \mathrm{O}, \mathrm{CH}_{3} \mathrm{OH}$ and $\mathrm{CH}_{3}$. It also formed positive ions at $\mathrm{m} / z$ 192, 163, 121, 119 and 107 in which ion of $\mathrm{m} / z 163$ is characteristic ion of $\mathrm{N}$-methylcoclaurine formed from ion of $\mathrm{m} / z 178$ due to loss of methyl group. Protonated parent ion $[\mathrm{M}+\mathrm{H}]^{+}$at $\mathrm{m} / z 295$ resolved at $30.2 \pm 0.2$ min was identified as isotanshinone II as it afforded mass fragments at $m / z 277\left[\mathrm{M}-\mathrm{H}_{2} \mathrm{O}\right]^{+}, 267[\mathrm{M}-\mathrm{CO}]^{+}, 249\left[\mathrm{M}-\mathrm{H}_{2} \mathrm{O}-\mathrm{CO}\right]^{+}$, $239\left[-\mathrm{C}_{3} \mathrm{H}_{4} \mathrm{O}\right]^{+}, 187,159$, and 111. Parent ion $[\mathrm{M}+\mathrm{H}]^{+}$of $\mathrm{m} / z 301$ showed predominant product ions at $\mathrm{m} / \mathrm{z}$ $283\left[\mathrm{M}-\mathrm{H}_{2} \mathrm{O}\right]^{+}$, and other ions at $m / z 269,257,229,137,129$ that match with standard spectra of peonidin. Protonated molecule $[\mathrm{M}+\mathrm{H}]^{+}$at $\mathrm{m} / z 714.264$ showed product ions at $\mathrm{m} / z 369,351,337,329$ and 311 , the characteristic ions of 5-allyloxysalvigenin, hence compound was identified as derivative of 5-allyloxysalvigenin.

Other up-regulated compounds whose level was found to be $>2$ fold but $<3$ fold were tinosporaside, jatrorrhizine, glucoside 493, 1-\{hydroxy[2-(trimethylammonio) ethyl]amino\}-1-oxo-2-dodecanaminium, 6-methylcoumarin, and cinnamaldehyde (Table 3). A protonated molecule $[\mathrm{M}+\mathrm{H}]^{+}$at $\mathrm{m} / z 500.169$ resolved at $18.5 \mathrm{~min}$ was identified as tinosporaside due the presence of dominant positive product ions at $\mathrm{m} / z 339$ [M-162] due loss of glucose moiety, 323 due loss of $\mathrm{H}_{2} \mathrm{O}$ and other ion at $m / z 317,207,137$ and 121 . The $[\mathrm{M}+\mathrm{H}]^{+}$ion of Jatrorrhizine was observed at $\mathrm{m} / z 338.1387$ and RT $28.8 \pm 0.2$. It produced fragment ions at $\mathrm{m} / z 323, \mathrm{~m} / z 308$, $\mathrm{m} / z 295$ and $m / z 294$ due to sequential loss of $\left[\mathrm{M}-\mathrm{CH}_{3}\right]^{+},\left[\mathrm{M}-\mathrm{CH}_{3}-\mathrm{CH}_{3}\right]^{+},\left[\mathrm{M}-\mathrm{CH}_{3}-\mathrm{CO}\right]^{+}$and $\left[\mathrm{M}-\mathrm{CH}_{4}-\mathrm{CO}\right]^{+}$ ions. The presence of $\left[\mathrm{M}-\mathrm{CH}_{3}-\mathrm{H}\right]^{+}$ions at $m / z 322$ confirm the presence of methoxyl groups at C9-C10 positions (Fig. S4). The $[\mathrm{M}+\mathrm{H}]^{+}$ion at $\mathrm{m} / z 655$ was resolved at $21.7 \mathrm{~min}$ and identified as unknown glucoside, as it afforded dominant ion at $\mathrm{m} / z 493$ due to neutral loss of $162 \mathrm{Da}$ of glucose moiety. Precursor ion $\mathrm{m} / z 161$ generate products at $\mathrm{m} / z 133$ and 105 due to consecutive loss of CO, hence presumed as 6-methylcoumarin. Cinnamaldehyde was identified as precursor ion $[\mathrm{M}+\mathrm{H}]^{+}$at $m / z 133$ forms product ions at $m / z 115$ and 105 due to loss of $\mathrm{H}_{2} \mathrm{O}$ and $\mathrm{CO}$. 


\begin{tabular}{|c|c|c|c|}
\hline Groups & Compound/Mass & Mass & $\begin{array}{l}\text { Retention } \\
\text { Time }\end{array}$ \\
\hline \multirow{2}{*}{ AIN } & Unidentified terpenoid & 457.36 & 41.69 \\
\hline & 8-Hydroxytinosporide & 390.1636 & 20.72 \\
\hline \multirow{6}{*}{$\mathrm{CON}$} & 952.7696 & 952.7696 & 41.34 \\
\hline & 5-Allyloxysalvigenin & 368.1217 & 20.66 \\
\hline & trans-Farnesol & 222.0835 & 6.37 \\
\hline & 636.6422 & 636.6422 & 41.28 \\
\hline & Reticuline (BP $m / z$ 151) & 329.1589 & 10.45 \\
\hline & N-Isovaleroylglycine & 159.0412 & 8.05 \\
\hline \multirow{5}{*}{ ANI } & 775.2589 & 775.2589 & 41.47 \\
\hline & 794.6717 & 794.6717 & 41.77 \\
\hline & Palmatoside C & 636.6416 & 41.47 \\
\hline & $(1,4)$ - $\alpha$-D-glucan & 180.0703 & 6.339 \\
\hline & 5-Aminovaleric acid & 117.0381 & 24.01 \\
\hline \multirow{5}{*}{ ALC } & 871.8031 & 871.8031 & 41.10 \\
\hline & 389.1615 & 389.1615 & 20.99 \\
\hline & 108.0251 & 108.0251 & 6.37 \\
\hline & 604.2386 & 604.2386 & 24.83 \\
\hline & Tinocordifolioside & 412.1304 & 30.13 \\
\hline \multirow{7}{*}{ ALL } & 5,6-Dihydrouracil & 114.0181 & 8.06 \\
\hline & Tinoridine & 315.216 & 25.16 \\
\hline & 614.4143 & 614.4143 & 28.46 \\
\hline & Tinosporinone & 329.1605 & 10.26 \\
\hline & Baenzigeroside A & 520.3456 & 25.5 \\
\hline & Tinosinen & 504.1888 & 6.38 \\
\hline & 110.0407 & 110.0407 & 6.44 \\
\hline \multirow{3}{*}{ TMI } & 11-Hydroxymustakone & 234.1723 & 27.30 \\
\hline & 794.6717 & 794.6717 & 41.77 \\
\hline & 880.6993 & 880.6993 & 41.41 \\
\hline
\end{tabular}

Table 2. Metabolites specifically and significantly present in various groups and represented in Venn diagram.

Up-regulated metabolites having fold change $<2.0$ as compared to control but $>2.0$ as compared to other groups were $\beta, 5 \alpha, 14 \alpha$-trihydroxyergosta-7,22-dien-6-one, cycloeucalenol, oblongine, 20-hydroxyecdysone, phenylmethanethiol, and cycloartane-24,25-diol-3-one (Table 3). The precursor ion scan and/or the low-energy CID-MS/MS of the protonated molecule at $\mathrm{m} / z 445$ (precursor ion) afforded the following products ion at $\mathrm{m} / \mathrm{z}$ $426\left[\mathrm{M}-\mathrm{H}_{2} \mathrm{O}\right]^{+}, 411\left[\mathrm{M}-\mathrm{H}_{2} \mathrm{O}-\mathrm{CH}_{3}\right]^{+}, 408\left[\mathrm{M}-2 \mathrm{H}_{2} \mathrm{O}\right]^{+}, 393\left[\mathrm{M}-2 \mathrm{H}_{2} \mathrm{O}-\mathrm{CH}_{3}\right]^{+} \cdot, 390\left[\mathrm{M}-3 \mathrm{H}_{2} \mathrm{O}\right]^{+}$that lead to its identification as $\beta, 5 \alpha, 14 \alpha$ - trihydroxyergosta-7,22-dien-6-one. The protonated molecule $[\mathrm{M}+\mathrm{H}]^{+}$at $\mathrm{m} / z 427$ afforded product ions at $m / z 409,393,383,367,353,343,325,301,233$ and 125, when matched with standard library it was identified as cycloeucalenol with a confidence score of 97.6. Precursor ion $[\mathrm{M}+\mathrm{H}]^{+}$at $m / z 315$ breakdown into positive product ion at $\left.297\left[\mathrm{M}-\mathrm{H}_{2} \mathrm{O}\right]^{+}, 287[\mathrm{M}-\mathrm{CO}]^{+}\right], 271[\mathrm{M}-26 \mathrm{Da}$ aromatic character], 257, 206, 178, 119 and 107. These product ions matched with standard spectra of oblongine with confidence score 93. The precursor ion $[\mathrm{M}+\mathrm{H}]^{+}$at $m / z 480$ resolved at 25.9 min was identified as 20 -hydroxyecdysone due to presence of product ions at $\mathrm{m} / z 462$ and 444 formed due to loss of $\mathrm{H}_{2} \mathrm{O}$ molecules, and other characteristic ions at $\mathrm{m} / z$ $429,426,411,408,393,363,345,344,328,327,300,145$, and 143 . The parent ion $[\mathrm{M}+\mathrm{H}]^{+}$at $m / z 125$ showed fragment ion at $m / z 109$ and identified as phenylmethanethiol. Precursor ion $[\mathrm{M}+\mathrm{H}]^{+}$at $m / z 459$ resolved at 26.5 min afforded product ions at $\mathrm{m} / z$ 441, 423 (due consecutive loss of $\mathrm{H}_{2} \mathrm{O}$ molecules), 313 and 175 as described earlier and identified as cycloartane-24,25-diol-3-one ${ }^{19}$.

Palmarin, feruloyltyramine, betaine, 1,3 dimethylpteridine-2,4-dione, sodium thiosalicylate, haplopine, isoquinolone alkaloid, amino-tridecanoic acid, glucoside of 207, tetramethylazobenzene, scalar-17(25)-en-19-ol were identified metabolite having fold change $<1.5$ as compared to control but have significant differential levels when compared to other groups (Table 3). The precursor ion scan of the protonated molecule at $\mathrm{m} / z 375$ (precursor ion) afforded the following products ion at $\mathrm{m} / z 357\left[\mathrm{M}-\mathrm{H}_{2} \mathrm{O}\right]^{+},[\mathrm{M}-\mathrm{CO}]^{+}, 329\left[\mathrm{M}-\mathrm{CO}, 2 \mathrm{H}_{2} \mathrm{O}\right]^{+}, 307,279$, 165 , and 125 that lead to its identification as palmarin. It was verified from Metlin standard database. Protonated molecule $[\mathrm{M}+\mathrm{H}]^{+}$resolved at $25.4 \mathrm{~min}$ of $\mathrm{m} / z 314$ was identified as feruloyltyramine when compared with synthetic standard library as it yielded the fragment ions at $m / z 296\left[\mathrm{M}-\mathrm{H}_{2} \mathrm{O}\right]^{+}, 284,270$. It yielded characteristic ion of primary acylium at $m / z 177$ which, further yielded fragment ions at $m / z 149,145$, and 117 . Betaine $(\mathrm{m} / z 118)$ was identified as produced identical spectra with synthetic betaine and resolution at $6.77 \mathrm{~min}$. The precursor ion scan and/or the low-energy CID-MS/MS of the protonated precursor ion at $m / z 193$ resolved at 24 min afforded

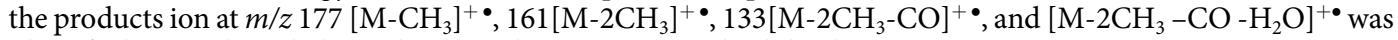
identified as 1,3 dimethylpteridine-2,4-dione using standard database. Parent ion $[\mathrm{M}+\mathrm{H}]^{+}$at $m / z 177.99$ was identified as sodium thiosalicylate as it yielded product ion at $\mathrm{m} / z$ 137, 133 and 105 using the standard database. 


\begin{tabular}{|c|c|c|c|c|c|c|c|c|c|c|}
\hline S. No. & Tentative Identification & RT & Mass & $\mathbf{p}$ (Corr) & AIN & ALC & ALL & ANI & FBG & TMI \\
\hline 1 & Choline* & 7.08 & 104.1070 & $6.79 \mathrm{E}-07$ & -1.8 & -1.4 & -6.2 & -3.7 & 1.1 & -1.3 \\
\hline 2 & Aminophenol* & 7.44 & 109.0571 & $2.59 \mathrm{E}-06$ & -1.1 & 1.3 & -2.5 & 1.1 & 3.1 & -2.5 \\
\hline 3 & Betaine & 6.77 & 117.0837 & $1.72 \mathrm{E}-05$ & 1.3 & -2.2 & -1.2 & -5.4 & -2.2 & -3.3 \\
\hline 4 & Phenylmethanethiol & 7.10 & 124.0332 & $7.74 \mathrm{E}-15$ & 1.4 & 1.0 & -4.8 & -3.8 & -1.1 & -1.8 \\
\hline 5 & Cinnamaldehyde & 24.0 & 132.0624 & $2.25 \mathrm{E}-11$ & 2.1 & -1.2 & -3.6 & -3.4 & -1.0 & 1.1 \\
\hline 6 & Isoquinoline $\mathrm{N}$-oxide & 8.72 & 145.0914 & $6.23 \mathrm{E}-09$ & -1.7 & 1.7 & -1.1 & -3.3 & 2.8 & 1.4 \\
\hline 7 & 6-Methylcoumarin* & 23.9 & 160.0583 & $3.46 \mathrm{E}-12$ & 2.1 & -1.2 & -3.2 & -3.8 & -1.2 & 1.1 \\
\hline 8 & Sodium thiosalicylate* & 7.10 & 176.9952 & $2.40 \mathrm{E}-12$ & 1.2 & -1.0 & -5.2 & -4.1 & -1.2 & -1.8 \\
\hline 9 & Acetamido-6-aminohexanoic acid & 27.9 & 189.1617 & $7.70 \mathrm{E}-09$ & -1.8 & -5.8 & -4.4 & -7.2 & -2.2 & -2.7 \\
\hline 10 & 1,3 Dimethylpteridine-2,4-dione & 24.0 & 192.0856 & $1.40 \mathrm{E}-14$ & 1.2 & -1.9 & -4.4 & -5.5 & -1.8 & -1.1 \\
\hline 11 & $\begin{array}{l}\text { 3-[4-(3-Aminopropylamino) butylamino] } \\
\text { propanoicacid }\end{array}$ & 28.7 & 217.2119 & $2.06 \mathrm{E}-13$ & -1.1 & -3.0 & -2.9 & -6.9 & -1.1 & -2.9 \\
\hline 12 & Ethyl ferulate & 41.4 & 222.0645 & $8.38 \mathrm{E}-07$ & -2.0 & -3.2 & -4.3 & -6.8 & -1.1 & -1.6 \\
\hline 13 & Amino-tridecanoic acid* & 34.9 & 229.2490 & $5.89 \mathrm{E}-09$ & 1.2 & -1.9 & -2.8 & -6.6 & -1.0 & -1.9 \\
\hline 14 & Glycosminine & 26.4 & 236.0925 & $1.26 \mathrm{E}-12$ & -1.3 & 1.0 & -4.3 & -5.9 & -1.2 & -1.5 \\
\hline 15 & Haplopine* & 31.4 & 245.2444 & $2.28 \mathrm{E}-09$ & 1.2 & -1.7 & -2.0 & -5.8 & 1.3 & -2.5 \\
\hline 16 & Trimethyldecahydrophenanthren-2-ol & 25.8 & 248.1147 & $1.30 \mathrm{E}-08$ & 1.7 & 2.4 & -2.8 & -2.3 & 1.8 & 1.2 \\
\hline 17 & Tinocordifolin & 26.2 & 250.1662 & $2.83 \mathrm{E}-09$ & -3.2 & -4.2 & -5.2 & -3.3 & 1.0 & -3.9 \\
\hline 18 & Chrysin* & 33.1 & 254.1615 & $2.22 \mathrm{E}-08$ & 1.7 & -2.5 & -5.4 & -7.4 & -1.5 & -2.3 \\
\hline 19 & Palmitic amide & 34.8 & 255.2656 & $1.41 \mathrm{E}-10$ & 1.4 & -1.0 & -2.0 & -5.1 & 1.7 & -1.0 \\
\hline 20 & Amylp-butylaminobenzoate & 31.7 & 263.1830 & $1.88 \mathrm{E}-08$ & -1.7 & -3.2 & -5.7 & -6.5 & -1.4 & -2.4 \\
\hline 21 & Neocryptotanshinone II & 28.2 & 270.1381 & $1.93 \mathrm{E}-11$ & 1.1 & 1.3 & -3.8 & -3.6 & 1.5 & -1.4 \\
\hline 22 & 13-Methyl-17-norabieta-15-ene-8-ylium & 34.7 & 273.2779 & $3.18 \mathrm{E}-09$ & 1.5 & -1.4 & -2.4 & -5.6 & 1.2 & -1.5 \\
\hline 23 & Alkaloid & 26.4 & 281.0918 & $3.02 \mathrm{E}-15$ & -1.4 & 1.0 & -4.3 & -5.9 & -1.2 & -1.5 \\
\hline 24 & Carboxylic acid & 25.6 & 281.1161 & $1.37 \mathrm{E}-06$ & 1.4 & -3.1 & -1.5 & -2.4 & 2.5 & 1.2 \\
\hline 25 & Coclaurine & 24.8 & 285.1466 & $3.04 \mathrm{E}-15$ & -1.6 & -7.8 & -1.9 & -3.1 & 1.0 & -1.4 \\
\hline 26 & Magnoflorine- $\left[\left(\mathrm{CH}_{3}\right) 2 \mathrm{NH}\right]$ & 26.4 & 296.1156 & $8.59 \mathrm{E}-16$ & -1.6 & -1.0 & -4.6 & -6.3 & -1.3 & -1.5 \\
\hline 27 & N-Methylcoclaurine & 24.4 & 299.1643 & $6.49 \mathrm{E}-14$ & 6.3 & 2.4 & 1.8 & 1.4 & 6.0 & 3.8 \\
\hline 28 & Unknown ester (Floridimine type) & 24.1 & 301.2001 & $1.65 \mathrm{E}-10$ & -3.3 & -3.5 & -4.5 & -9.5 & -2.1 & -2.5 \\
\hline 29 & Sphinganine & 38.1 & 301.3094 & $5.93 \mathrm{E}-05$ & 3.2 & 1.6 & -1.2 & -2.9 & 1.8 & 1.1 \\
\hline 30 & $(-)-$ Gallocatechin & 8.03 & 306.0222 & $5.72 \mathrm{E}-05$ & -1.2 & -1.6 & -6.0 & -4.6 & -1.7 & -3.3 \\
\hline 31 & 3-Oxo-nonadecanoic acid & 41.4 & 312.2785 & $1.68 \mathrm{E}-10$ & -3.8 & -6.0 & -5.3 & -5.4 & -1.6 & -2.2 \\
\hline 32 & Feruloyltyramine* & 25.4 & 313.1428 & $1.84 \mathrm{E}-14$ & 1.3 & -3.2 & -3.3 & -5.2 & -1.1 & -2.5 \\
\hline 33 & $(+/-)$ Oblongine* & 27.0 & 313.1793 & $2.99 \mathrm{E}-13$ & 1.8 & -2.8 & -2.3 & -2.1 & 1.9 & -2.5 \\
\hline 34 & $(+/-)$ Oblongine & 25.6 & 313.1793 & $5.95 \mathrm{E}-14$ & -1.3 & -2.1 & -5.1 & -6.7 & -2.5 & -1.3 \\
\hline 35 & Phytosphingosine & 34.8 & 317.3048 & $3.61 \mathrm{E}-15$ & 2.1 & 2.1 & 1.3 & -3.2 & 2.7 & 1.9 \\
\hline 36 & Robinobiose & 27.0 & 326.1280 & $3.00 \mathrm{E}-11$ & -2.0 & -2.1 & -4.4 & -3.1 & -9.4 & -2.3 \\
\hline 37 & Dideoxysulphonated steroid & 24.3 & 326.2064 & $7.72 \mathrm{E}-07$ & -1.8 & -2.6 & -4.1 & -5.8 & -1.0 & -1.0 \\
\hline 38 & Icosasphinganine & 41.0 & 329.3416 & $2.31 \mathrm{E}-05$ & 1.6 & 1.2 & -1.9 & -4.7 & 1.4 & 1.0 \\
\hline 39 & Jatrorrhizine* & 28.8 & 337.1444 & $3.71 \mathrm{E}-17$ & -1.3 & -1.5 & -3.8 & -8.3 & -1.7 & -1.8 \\
\hline 40 & Magnoflorine $[\mathrm{M}+]$ & 26.4 & 342.1712 & $2.78 \mathrm{E}-14$ & -1.5 & 1.0 & -4.5 & -6.1 & -1.2 & -1.5 \\
\hline 41 & 8-Oxoberberine & 29.8 & 351.1594 & $1.97 \mathrm{E}-15$ & -1.6 & 1.0 & -3.6 & -6.0 & -1.2 & 1.0 \\
\hline 42 & Palmatine* & 32.4 & 352.2358 & $1.64 \mathrm{E}-08$ & -3.6 & 2.1 & -3.4 & -2.1 & -1.0 & -1.8 \\
\hline 43 & Corydine methyl ether & 27.4 & 355.1914 & $3.48 \mathrm{E}-11$ & -2.7 & -4.4 & -3.0 & -7.9 & -1.2 & -2.3 \\
\hline 44 & N-Tetrahydropalmatine* & 29.0 & 356.1393 & $2.21 \mathrm{E}-09$ & -1.5 & -1.1 & -5.9 & -5.2 & -2.1 & -3.0 \\
\hline 45 & Isocorydine- $\mathrm{N}$-oxide & 25.6 & 357.1709 & $2.52 \mathrm{E}-11$ & 1.0 & -1.3 & -4.6 & -4.3 & 1.3 & -1.5 \\
\hline 46 & Isoquinolone alkaloid & 26.8 & 357.2074 & $3.19 \mathrm{E}-09$ & 1.2 & -1.9 & -3.1 & -2.2 & 2.8 & 1.0 \\
\hline 47 & Tinosporin & 27.1 & 358.1552 & $6.08 \mathrm{E}-10$ & -2.5 & -2.1 & -6.8 & -5.4 & -6.0 & -3.0 \\
\hline 48 & Glucoside & 17.9 & 368.1221 & $3.72 \mathrm{E}-08$ & 1.2 & -5.4 & -1.1 & -3.8 & -1.2 & -1.3 \\
\hline 49 & Pentamethoxyflavone & 28.4 & 372.1349 & $2.25 \mathrm{E}-14$ & -3.9 & 1.0 & -6.3 & -3.8 & -2.4 & -1.9 \\
\hline 50 & Palmarin & 41.8 & 374.2572 & $7.34 \mathrm{E}-06$ & 1.4 & -1.8 & -3.2 & -5.7 & -1.3 & -1.3 \\
\hline 51 & Steroidal Compound & 24.7 & 387.2611 & $1.55 \mathrm{E}-07$ & -2.2 & -2.5 & -3.5 & -6.7 & -1.2 & 1.0 \\
\hline 52 & Salvinorin B & 28.5 & 390.1472 & $7.17 \mathrm{E}-11$ & -6.7 & -2.1 & -6.6 & -4.8 & -3.6 & -2.5 \\
\hline 53 & Stigmastan-3,5-diene & 41.7 & 396.3033 & $1.98 \mathrm{E}-07$ & -1.5 & -2.8 & -4.4 & -7.2 & -1.3 & -1.7 \\
\hline 54 & Tinocordifolioside & 26.1 & 412.2255 & $3.53 \mathrm{E}-10$ & -3.0 & -4.8 & -4.0 & -2.4 & 1.3 & -3.4 \\
\hline 55 & Lycopene derivative & 35.7 & 414.2205 & $5.05 \mathrm{E}-09$ & -1.6 & -2.7 & -4.8 & -6.9 & -1.2 & -1.8 \\
\hline 56 & Cycloeucalenol & 25.9 & 426.2928 & $5.57 \mathrm{E}-16$ & 1.8 & -2.3 & -5.3 & -1.8 & 1.0 & -1.4 \\
\hline
\end{tabular}




\begin{tabular}{|l|l|l|l|l|r|r|r|r|r|r|}
\hline S. No. & Tentative Identification & RT & Mass & p (Corr) & AIN & ALC & ALL & \multicolumn{1}{|c|}{ ANI } & FBG & TMI \\
\hline 57 & Glucoside & 24.5 & 442.2119 & $5.21 \mathrm{E}-14$ & -4.5 & -1.9 & -3.1 & -6.8 & -2.5 & -6.7 \\
\hline 58 & $33,5 \alpha, 14 \alpha-$ Trihydroxyergosta-7,22-dien-6-one & 25.9 & 444.3046 & $1.02 \mathrm{E}-16$ & 1.8 & -2.3 & -5.4 & -1.7 & 1.0 & -1.4 \\
\hline 59 & Glucoside of m/z 286 & 23.4 & 447.2062 & $6.22 \mathrm{E}-15$ & 2.8 & -1.4 & 1.4 & -1.9 & 4.2 & 1.5 \\
\hline 60 & Cycloartane-24,25-diol-3-one & 26.5 & 458.3206 & $1.17 \mathrm{E}-11$ & 1.4 & -1.9 & -6.6 & -1.3 & -2.4 & -2.6 \\
\hline 61 & 20-Hydroxyecdysone* & 25.9 & 480.3273 & $6.75 \mathrm{E}-14$ & 1.7 & -2.3 & -5.4 & -1.8 & 1.0 & -1.4 \\
\hline 62 & Auricularine & 26.5 & 494.3421 & $4.19 \mathrm{E}-08$ & -1.2 & -2.1 & -8.6 & -1.8 & -2.7 & -2.9 \\
\hline 63 & Tinosporaside & 18.5 & 500.1694 & $4.34 \mathrm{E}-12$ & 2.5 & 4.5 & 1.7 & -1.4 & 3.8 & 3.7 \\
\hline 64 & Baenzigeroside A & 25.2 & 520.3468 & $4.63 \mathrm{E}-11$ & -2.9 & -2.9 & -5.4 & -8.1 & -1.6 & -3.3 \\
\hline 65 & O-Glucoside derivative & 28.0 & 542.2257 & $4.87 \mathrm{E}-10$ & -3.5 & -2.1 & -1.6 & -5.5 & 1.5 & -1.8 \\
\hline 66 & O-Glucoside derivative & 25.2 & 560.2090 & $2.31 \mathrm{E}-11$ & -3.5 & -5.3 & -3.8 & -8.8 & -2.9 & -5.1 \\
\hline 67 & Isotanshinone II $*$ & 30.2 & 294.1231 & 0.048351 & 3.3 & 5.4 & 3.4 & 5.2 & 2.9 & 3.2 \\
\hline 68 & Cordifolide A & 26.9 & 598.4068 & $2.64 \mathrm{E}-09$ & -1.5 & -7.3 & -2.9 & -4.5 & -1.3 & -2.0 \\
\hline 69 & Isoquinoline alkaloid & 41.5 & 616.4461 & $2.59 \mathrm{E}-04$ & -1.7 & -3.4 & -5.2 & -5.8 & -1.3 & -2.2 \\
\hline 70 & Saponin glycoside & 25.6 & 620.2330 & $5.25 \mathrm{E}-09$ & -2.3 & -2.6 & -6.1 & -7.8 & -1.9 & -2.6 \\
\hline 71 & Chrysoeriol C-hexoside O-hexoside & 20.8 & 624.2278 & 0.034911 & -1.0 & -1.1 & -3.8 & -2.4 & 1.8 & 1.0 \\
\hline 72 & Unknown cinnamic derivative & 41.4 & 633.4716 & $2.54 \mathrm{E}-07$ & -2.6 & -3.9 & -5.8 & -6.0 & -1.3 & -2.8 \\
\hline 73 & Glucoside of m/z 493 & 21.7 & 654.2404 & $4.05 \mathrm{E}-07$ & 2.2 & -1.4 & -1.0 & -1.5 & 4.9 & 1.0 \\
\hline 74 & 5-Allyloxysalvigenin derivative & 17.8 & 714.2648 & $1.18 \mathrm{E}-09$ & 1.9 & -1.3 & -3.5 & -4.1 & 1.0 & -1.2 \\
\hline 75 & Unknown of $m / z 184.08$ & 41.2 & 785.6281 & 0.001743 & -1.3 & -2.6 & -3.8 & -2.0 & 1.9 & 1.0 \\
\hline 76 & $\begin{array}{l}\text { Diosgenin 3-[glucosyl-(1->4)-rhamnosyl-(1->4)- } \\
\text { [rhamnosyl-(1->2)]-glucoside }\end{array}$ & 23.9 & 1030.3995 & $3.42 \mathrm{E}-13$ & -1.4 & -3.3 & -4.4 & -5.8 & -2.6 & 1.0 \\
\hline
\end{tabular}

Table 3. Fold change found in the metabolites in different groups in comparison to control [CON] group. Compounds have been identified using mass error $>5 \mathrm{ppm}$ and fragment ions and arranged according to their retention time. Note: *Compound identified with standard compound; Italics: Compounds identified by structure elucidation with help of MSC (Agilent Technologies) and Metfrag software; all other compounds were identified by comparing MS/MS spectra with standard libraries from METLIN and MassBank. Unidentified compounds are represented as unknown or by formula.

Protonated molecule $[\mathrm{M}+\mathrm{H}]^{+}$at $m / z 246$ resolved at $31.4 \mathrm{~min}$ and yielded product ions at $m / z 228\left[\mathrm{M}-\mathrm{H}_{2} \mathrm{O}\right]^{+}$, $218[\mathrm{M}-\mathrm{CO}]^{+}, 214,202,186,174$ and 142 matched with haplopine in standard database Metlin with a confidence score of 97.23 . Precursor ion $[\mathrm{M}+\mathrm{H}]^{+}$at $\mathrm{m} / z 358$ resolved at $26.8 \mathrm{~min}$ afforded characteristic fragments ions at $\mathrm{m} / z 192,151$ and 137 of isoquinolone, however it could not be identified hence, named as isoquinolone alkaloid. Precursor ion $[\mathrm{M}+\mathrm{H}]^{+}$at $\mathrm{m} / z 230$ yielded product ions of 212 and 195 due neutral loss of $\mathrm{H}_{2} \mathrm{O}$ followed by $\mathrm{NH}_{4}$. It also yielded characteristic ions of $\mathrm{m} / z$ 184, 167, 155 related to tridecanoic acid; hence it was identified as amino-tridecanoic acid. A precursor ion $[\mathrm{M}+\mathrm{H}]^{+}$at $\mathrm{m} / z 369$ afforded product at $\mathrm{m} / z 207$ due to loss of glucose moiety, further identification could not be established, hence named as glucoside of 207. Parent ion $[\mathrm{M}+\mathrm{H}]^{+}$at $\mathrm{m} / z 358$ resolved at $25.6 \mathrm{~min}$ yielded product ions at $\mathrm{m} / z 343\left[\mathrm{M}-\mathrm{CH}_{3}\right]^{+}, 327\left[\mathrm{M}-2 \mathrm{CH}_{3}\right]^{+} \bullet, 272,259,191,163,137$, and 123, hence, identified as scalar-17(25)-en-19-ol as reported previously ${ }^{20}$.

Differential metabolites in ALC group. In this group, compounds of $108,389,604,871 \mathrm{Da}$ and tinocordifolioside were identified exclusively. Precursor ion scan of the protonated molecule at $\mathrm{m} / z 413$ afforded the products ion at $m / z 251$ die loss of $162 \mathrm{Da}$ (glucose moiety), hence identified as tinocordifolioside. Other compounds uniquely present in this group could not be identified. ALC group also showed elevated levels $(>2.0$ fold) of isotanshinone IIA, tinosporaside, saponin, N-methylcoclaurine (described in AIN group), dehydrocorybulbine, and palmatine (Table 3). The compound resolved at $32.4 \mathrm{~min}$ of $m / z 353[\mathrm{M}+\mathrm{H}]^{+}$yielded the fragment ions at $\mathrm{m} / z 337\left[\mathrm{M}-\mathrm{CH}_{4}\right]^{+}, 321\left[\mathrm{M}-2 \mathrm{CH}_{4}\right]^{+}, 308,294$ and 279 was unambiguously assigned as dehydrocorybulbine after comparing with standard spectra. The $[\mathrm{M}+\mathrm{H}]^{+}$ion of palmatine was observed at $m / z 352$. Low-energy CID-MS/ MS afforded the prominent product at $\mathrm{m} / z 337\left[\mathrm{M}-\mathrm{CH}_{3}\right]^{+} \cdot$ due to the loss of methyl radical. It produced ions product ions at $m / z 322$ and 308 due to sequential losses of hydrogen radical and CO. Product ions including others at $m / z 294,278,264$ and 250 were equivalents to mass spectra obtained from synthetic palmatine (Fig. S4), hence compound was identified as palmatine.

Compound found to present in higher amounts as compared to other groups but $<2.0$ fold as compared to CON group were (9Z,14Z)-octadeca-9,14-dien-6-ynoic acid, isoquinoline N-oxide, peonidin, N-[3(cyclohexylamino)-4-methoxyphenyl] acetamide, tetramethylazobenzene, aminophenol, borapetosides $\mathrm{D}$ and tetrahydro-4-acetyl-1-methyl-7-(4-pyridinyl)-3(2 H) isoquinolinone (Table 3). Precursor ion resolved at 8.72 and at $m / z 146[\mathrm{M}+\mathrm{H}]^{+}$yielded major product ion at $\mathrm{m} / z 129$ and 128 due to loss of $\mathrm{N}$ linked oxygen as $\mathrm{H}_{2} \mathrm{O}$ and 102 due Diels-Alder fragmentation, identified as isoquinoline $\mathrm{N}$-oxide. Aminophenol was identified using isomeric pattern of precursor ion keeping mass error $>5 \mathrm{ppm}$. Precursor ion $[\mathrm{M}+\mathrm{H}]^{+}$at $\mathrm{m} / z 699$ resolved at $27.4 \mathrm{~min}$ yielded product ion at $\mathrm{m} / z 537$ due loss of glucose moiety $(162 \mathrm{Da}$ ) that further yielded peak at $\mathrm{m} / z 121$ due to the furan ring conjunction indicated that these compounds are furanoid diterpenes with an oxygen at C-12 and an angular methyl group at C-9. It also afforded product ions at $m / z 375,357,343,265,231,205,185,159,149,121$ and 133 which led its identification as borapetoside D. 


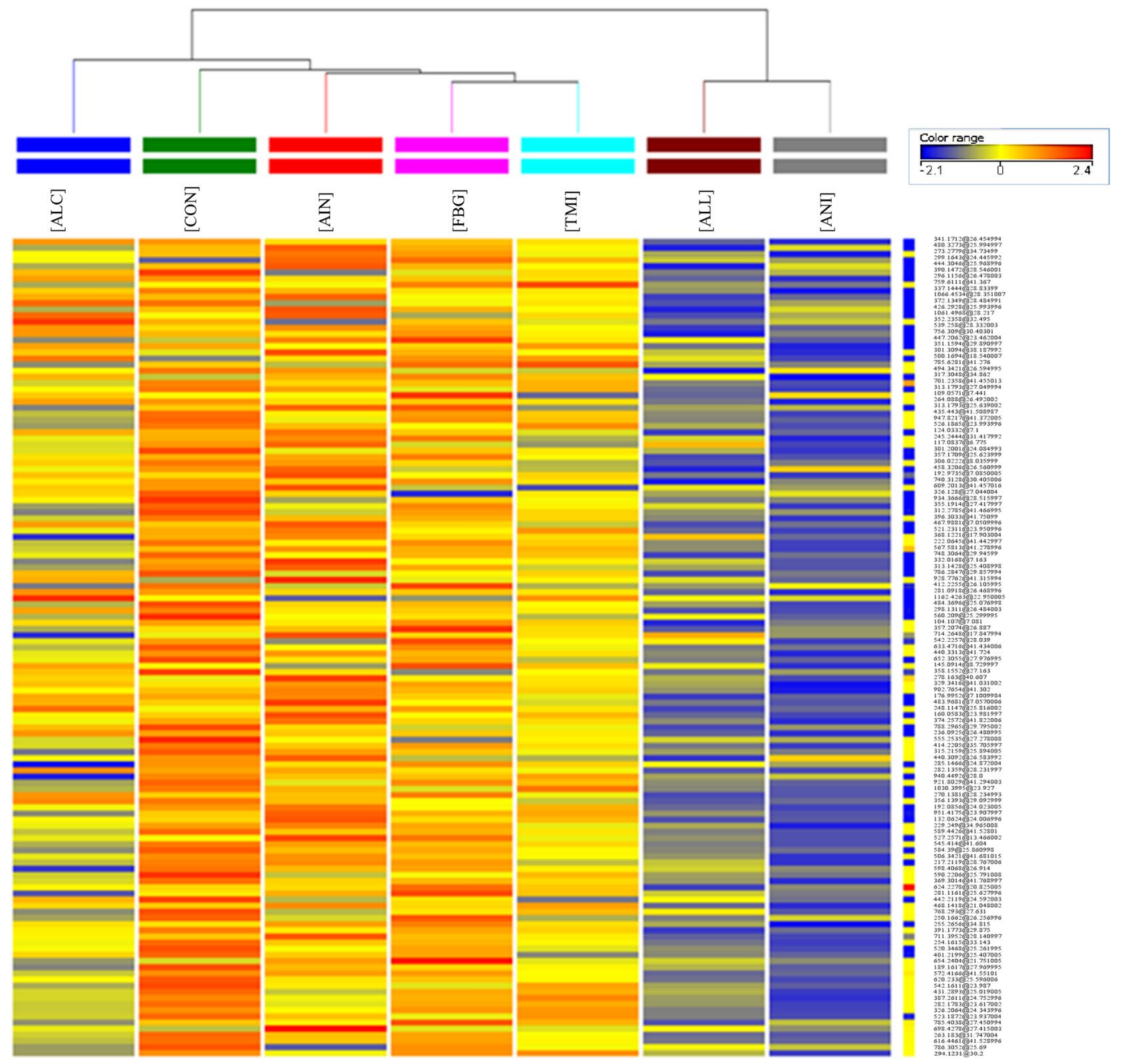

Figure 4. Spearman correlation heatmap of the metabolites without missing values, with compounds marked on the side of the map. Each row represents a sample and each column represents a metabolite feature. Color key indicates the normalized abundance of each metabolite expression value across the samples. The red and blue color indicates the highest and lowest metabolite expression values respectively. The figure clearly depicts the distinctness of ALL and ANI having lowest levels of most of the metabolites.

Differential metabolites in FBG group. Venn diagram analysis didn't show presence of any specific compound in FBG group. However, T. cordifolia supported by F. bengalensis found to have $>2.0$ fold increased level of $\mathrm{N}$-methylcoclaurine, glucosides of 493 and 286 Da compounds, tinosporaside, aminophenol, isotanshinone II, isoquinoline $\mathrm{N}$-oxide, isoquinolone alkaloid and one unknown carboxylic acid derivative (Table 3). Other identified compounds having levels $<2.0$ fold as compared to CON group but significant altered levels as compared to other groups includes oblongine, peonidin, chrysoeriol C-hexoside O-hexoside, palmitic amide, tetramethylazobenzene, 5-allyloxysalvigenin derivative, haplopine, tinocordifolioside, borapetoside D and choline (Table 3). The protonated molecule $[\mathrm{M}+\mathrm{H}]^{+}$observed at $\mathrm{m} / z 625$ corresponding to chrysoeriol C-hexoside O-hexoside as its fragment ions at 463 and 445 were assigned as loss of C-glucose and O-glucose moieties. Further, product ions at $\mathrm{m} / z$ 313, 295 and 163 confirmed its identity as chrysoeriol. Precursor ion $[\mathrm{M}+\mathrm{H}]^{+}$at $m / z 714$ resolved at 17.8 min afforded product ions at $\mathrm{m} / z 535,369,351,337,329$ and 311. Last four peaks were found to match with standard mass spectra of allyloxysalvigenin; hence it was named as allyloxysalvigenin derivative. Precursor ion resolved at $25.6 \mathrm{~min}$ and $[\mathrm{M}+\mathrm{H}]^{+}$at $m / z 358$ yielded product ions at $m / z 343\left[\mathrm{M}-\mathrm{CH}_{3}\right]^{+}, 313\left[\mathrm{M}-\mathrm{CH}_{3}-\mathrm{NH}_{2}\right]^{+}$, $295\left[-\mathrm{H}_{2} \mathrm{O}\right]^{+}, 281,263$ and 133, indicated presence of $\mathrm{N}$-methyl and however it could not be identified. Precursor ion $[\mathrm{M}+\mathrm{H}]^{+}$at $m / z 246$ resolve at 31.4 yielded product ions at $m / z 231\left[\mathrm{M}-\mathrm{CH}_{3}\right]^{+} \bullet, 230,228\left[\mathrm{M}-\mathrm{H}_{2} \mathrm{O}\right]^{+}, 216[\mathrm{M}$ $\left.-2 \mathrm{CH}_{3}\right]^{+} \bullet, 218,214,202,186$, and 174 was established as haplopine when compared with standard.

Differential metabolites in TMI group. 11-Hydroxymustakone $(m / z=235.1723)$ and two unknown compounds of $m / z 795$ and 881 were exclusively present in this group only. Precursor ion $[\mathrm{M}+\mathrm{H}]^{+}$at $m / z 235$ afforded product ions at $m / z 217\left[\mathrm{M}-\mathrm{H}_{2} \mathrm{O}\right]^{+}, 189\left[\mathrm{M}-\mathrm{H}_{2} \mathrm{O}-\mathrm{CO}\right]^{+}, 175\left[\mathrm{M}-\mathrm{C}_{3} \mathrm{H}_{6}\right]^{+} 161,131$ and 107 corresponding unambiguously to 11-hydroxymustakone when compared with standard spectra. T. cordifolia co-occurred with T. indica didn't show significant increased levels of metabolites (Table 2). However, isotanshinone IIA, tinosporaside and $\mathrm{N}$-methylcoclaurine were found to be increased as compared to control. Down-regulated 
metabolites includes cordifolide A, 3-oxo-nonadecanoic acid, glaucine, cyclohexane-1,3-dione, robinobiose, isonicotonic acid, amyl p-butylaminobenzoate, aminophenol, oblongine, haplopine, feruloyltyramine, (S)-6-O-methylnorlaudanosoline, sulphonated steroid, cycloartane-3-oxo-24,25-diol, phosphatidylcholine, myricetin, makisterone A, N-tetrahydropalmatine, tinosporin, palmatoside $\mathrm{C}$, gallocatechin, betaine, baenzigeroside $\mathrm{B}$, tinocordifolioside and tinocordifolin (Table 3).

Differential metabolites in ALL group. ALL group exclusively contains 5, 6-dihydrouracil, tinoridine, baenzigeroside A, tinosinen and unknown compounds at $\mathrm{m} / z 615$ and 111 . After initial analysis, 5,6 dihydrouracil was identified using mass range from $\mathrm{m} / z 50$ to 100 in Q-TOF-MS as it yielded characteristic product ions at $m / z 73,55$, and 56 due to loss of $\mathrm{H}_{2} \mathrm{CCO}, \mathrm{NH}_{2}, \mathrm{CONH}_{2}$ and formation of $\mathrm{NH}_{2} \mathrm{CONH}_{3}{ }^{+}$. Precursor ion $[\mathrm{M}+\mathrm{H}]^{+}$at $m / z 316$ yielded product ions at $271\left[\mathrm{M}-\mathrm{C}_{2} \mathrm{H}_{6} \mathrm{O}\right]^{+}, 198\left[\mathrm{M}-\mathrm{C}_{8} \mathrm{H}_{9} \mathrm{~N}\right]^{+}, 152\left[198-\mathrm{C}_{2} \mathrm{H}_{6} \mathrm{O}\right]^{+}$, and 120 $\left[\mathrm{M}-\mathrm{C}_{9} \mathrm{H}_{11} \mathrm{NO}_{2} \mathrm{~S}\right]^{+}$, hence structure established to tinoridine. Parent ion $[\mathrm{M}+\mathrm{H}]^{+}$at $\mathrm{m} / z 330$ yielded product ions at $m / z 314\left[\mathrm{M}-\mathrm{CH}_{3}\right]^{+}, 298\left[\mathrm{M}-2 \mathrm{CH}_{3}\right]+, 168$ and 151 , however structure could not be established. Precursor ion $[\mathrm{M}+\mathrm{H}]^{+}$at $m / z 521$ yielded product ions at $m / z 359$ [M -Glu], 313, 247, 213, 203, 165, 151, 133, 119, and 105 similar to baenzigeroside A, hence compound was established as baenzigeroside A. Precursor ion $[\mathrm{M}+\mathrm{H}]^{+}$ at $\mathrm{m} / z 505$ yielded product ions at $\mathrm{m} / z 373$ ad 343 due to loss of pentose and hexose moieties. Further ion at $\mathrm{m} / \mathrm{z}$ 343 yielded $325\left[\mathrm{M}-\mathrm{H}_{2} \mathrm{O}\right]^{+}, 311\left[\mathrm{M}-\mathrm{CH}_{3}\right]^{+}, 293\left[-\mathrm{H}_{2} \mathrm{O}\right], 275\left[-\mathrm{H}_{2} \mathrm{O}\right], 211,197,163$ and 133 unambiguously established the structure of tinosinen.

T. cordifolia grown on A. lebbeck (ALL) didn't show elevated levels of metabolites after elimination of the metabolites without missing values (Table 3). Isotanshinone IIA, N-methylcoclaurine, tinosporaside, borapetosides D were found to be up-regulated as compared with control. However, in comparison of other groups these compounds were under expressed in this group. In Table 3, most of the compounds were found to be significantly suppressed in T. cordifolia climbed on ALL, which is the highest number of suppressed metabolites among all the groups. Some of known metabolites i.e. haplopine, oblongine, cordifolide A, glaucine, 6-methylcoumarin, palmarin, feruloyltyramine, dehydrocorybulbine, palmatine, cinnamaldehyde, jatrorrhizine, magnoflorine, tanshindiol, tinocordifolin, cycloeucalenol, dideoxyecdysone, 20-hydroxyecdysone, palmatoside $\mathrm{C}, \mathrm{N}$-tetrahydropalmatine, tinosporin, and makisterone A were among the down-regulated metabolites in this group.

Differential metabolites in ANI group. In this group $\alpha$-D-glucan, 5-aminovaleric acid, Palmatoside C and two unidentified compounds at $m / z 775$ and 795 were found to be uniquely present. Precursor ion $[\mathrm{M}+\mathrm{H}]^{+}$ at $m / z 181$ showed product ions as of in glucose when compared standard, hence named $\alpha$-D-glucan (breakdown unit of glucans). Protonated molecule $[\mathrm{M}+\mathrm{H}]+$ at $\mathrm{m} / z 118$ produce product ions at $\mathrm{m} / z 101$ and 100 due to loss of $\mathrm{NH}_{3}$ and $\mathrm{H}_{2} \mathrm{O}$ molecules, therefore identified as 5-aminovaleric acid also confirmed using the monoisotopic mass error $<5 \mathrm{ppm}$. Biotic interactions of A. nilotica (ANI) with T. cordifolia displayed elevated levels of isotanshinone IIA and $(9 Z, 14 Z$ )-octadeca-9,14-dien-6-ynoic acid (Table 3). Most of the other metabolites were found to be down-regulated as in case of ALL group that includes oblongine, palmatine, tinocordifolin, cinnamaldehyde, feruloyltyramine, magnoflorine, and jatrorrhizine well-known metabolites from T. cordifolia.

Upregulated metabolites in CON group. Control group contain specific metabolites namely 5-allyloxysalvigenin, trans-farnesol, reticuline (BP $\mathrm{m} / z$ 151) and two unidentified metabolites of $\mathrm{m} / z 953$ and 637. Precursor ion $[\mathrm{M}+\mathrm{H}]^{+}$at $m / z 369$ resolved at 20.7 min yielded product ions at $353\left[\mathrm{M}-\mathrm{CH}_{4}\right]^{+}, 351\left[\left[\mathrm{M}-\mathrm{H}_{2} \mathrm{O}\right]^{+}\right.$, $321\left[\mathrm{M}-\mathrm{CH}_{3} \mathrm{OH}\right]^{+}, 323\left[\mathrm{M}-\mathrm{H}_{2} \mathrm{O}-\mathrm{CO}\right]^{+}, 311,297,285,269$ and 107, a typical fingerprint of allyloxysalvigenin. Parent ion $[\mathrm{M}+\mathrm{H}]^{+}$at $\mathrm{m} / z 223$ resolved at 6.4 min yielded product ion of $\mathrm{m} / z 205$ due to loss of water molecule, 163 due to loss of $\mathrm{CH}_{3} \mathrm{CH}=\mathrm{CH}_{2}, 149$ due to loss of $\mathrm{CH}_{4}, 135$ due to loss of $\mathrm{CH}_{3}, 123,121$ due to loss of $\mathrm{CH}_{3}$, 109 and 107 again due to loss of $\mathrm{CH}_{3}$ established its structure as trans-farnesol. Protonated molecule $[\mathrm{M}+\mathrm{H}]^{+}$ resolved at $10.5 \mathrm{~min}$ and at $\mathrm{m} / z 330$ afforded product ion at $\mathrm{m} / z 314,301,298,287,244,192,151,149,137$ and 123. These are the characteristic ion of reticuline. It was further confirmed by the presence of the most abundant ion of $\mathrm{m} / \mathrm{z} 192$ which formed due to loss of $138 \mathrm{Da}$ through four member ring arrangements and the ion of $\mathrm{m} / \mathrm{z}$ 137 formed due to benzylic cleavage.

A number of other metabolites were also found to be up-regulated in $\mathrm{CON}$ group as compared to other groups (Table 3) and some important ones are described here. 8-Oxoberberine $[\mathrm{M}+\mathrm{H}]^{+}$ion at $\mathrm{m} / z 352.1594$ was identified due to sequentially loss of $\mathrm{CH}_{3}, \mathrm{CO}$ and $\mathrm{CH}_{4}$ generated ions at $\mathrm{m} / z 337,308$ and 292. The precursor ion $[\mathrm{M}+\mathrm{H}]^{+}$at $m / z 356$ yielded product ions at $m / z 341\left[\mathrm{M}-\mathrm{CH}_{3}\right]^{+}, 326\left[\mathrm{M}-2 \mathrm{CH}_{3}\right]^{+}, 313\left[\mathrm{M}-\mathrm{CH}_{3}-\mathrm{CO}\right]^{+}, 311,279$, 265, 206, 192 [prominent peak due ring cleavage] and 133 has been identified as tetrahydropalmatine using reference standard. Another parent ion at $m / z 342[\mathrm{M}]^{+}$generated strong ions peak at $\mathrm{m} / z 297$ due to loss of dimethylamine molecule $\left[\left(\mathrm{CH}_{3}\right)_{2} \mathrm{NH}\right.$, at $\mathrm{m} / z 282,265$ and 237 due to loss of methyl radical, methanol and $\mathrm{CO}$, respectively. These are the characteristic feature of protoberberine alkaloids; hence compound was unambiguously identified as magnoflorine (Fig. S4). Two precursor ions $[\mathrm{M}+\mathrm{H}]^{+}$at $\mathrm{m} / z 297$ and 265 were identified as degradation products of magnoflorine, as these afforded the peaks of magnoflorine - $\left[\left(\mathrm{CH}_{3}\right)_{2} \mathrm{NH}\right]+$ and magnoflorine $-\left[\left(\mathrm{CH}_{3}\right)_{2} \mathrm{NH}\right.$ ] $\left.-\mathrm{CH}_{3} \mathrm{OH}-\mathrm{CH}_{3}\right]^{+}$. The precursor ions $[\mathrm{M}+\mathrm{H}]^{+}$at $\mathrm{m} / z 297$ generated product ions at $m / z 265,237,219,207$ and 179 and $[\mathrm{M}+\mathrm{H}]^{+} \mathrm{m} / z 265$ generated the product ions at $m / z 237,219,207$ and 179 as in case of magnoflorine. Precursor ion $[\mathrm{M}+\mathrm{H}]^{+}$at $\mathrm{m} / z 355$ generated product ions at $\mathrm{m} / z 311\left[\mathrm{M}-\mathrm{CO}_{2}\right]^{+}, 296\left[\mathrm{M}-\mathrm{CO}-\mathrm{CH}_{3}\right]^{+}, 279[\mathrm{~m} / z$ $\left.311-\mathrm{CH}_{3} \mathrm{OH}\right]^{+}, 265\left[-\mathrm{CH}_{3}\right]^{+}, 251\left[-\mathrm{CH}_{3}\right]^{+}, 229,215,192$, and 104, just like magnoflorine with addition methyl group, hence compound was identified as corydine methyl ether. Precursor ion $[\mathrm{M}+\mathrm{H}]^{+}$at $m / z 314$ resolved at 25.6 min yielded product ions at $m / z 299\left[\mathrm{M}-\mathrm{CH}_{3}\right]^{+}, 281\left[\mathrm{M}-\mathrm{CH}_{3}-\mathrm{H}_{2} \mathrm{O}\right]+, 269\left[\mathrm{M}-\left(\mathrm{CH}_{3}\right) 2 \mathrm{NH}\right]^{+}, 237[\mathrm{~m} / z$ $\left.269-\mathrm{CH}_{3} \mathrm{OH}\right]^{+}, 209,192$ [M- Isoquinoline], $175\left[\mathrm{~m} / z 269-\mathrm{C}_{6} \mathrm{H}_{6}\right.$ ], 143, 121, 107 [Benzylic cleavage], hence, it was identified as oblongine. Protonated molecule $[\mathrm{M}+\mathrm{H}]^{+}$at $\mathrm{m} / z 358$ resolved at 26.8 min generated products

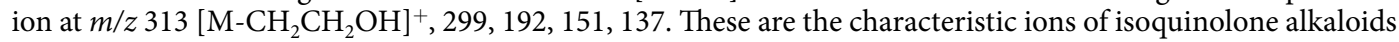


as the product ion at $m / z 299$ formed by loss of $\mathrm{CH}_{3} \mathrm{NH}_{2}, \mathrm{~m} / z 192$ and 137 represent the isoquinoline and the benzylic cleavage fragment as discussed in case of oblongine, hence compound was named isoquinoline alkaloid. Precursor ion $[\mathrm{M}+\mathrm{H}]^{+}$at $m / z 295$ generated ion $m / z 277,253,249$ and 221 due to loss of $\mathrm{H}_{2} \mathrm{O}, \mathrm{CH}=\mathrm{CHCH}_{3}$, $\mathrm{H}_{2} \mathrm{O}$ and $\mathrm{CO}$, was identified as isotanshinone IIA by comparing with standard spectra. Structure of precursor ion $[\mathrm{M}+\mathrm{H}]^{+}$at $m / z 702$ resolved at $41.4 \mathrm{~min}$ could not be established but it afforded product ions at $\mathrm{m} / z 563$, $445,429,371,355,341,297,281,267,223,207$ and 149, later one are similar ions to tanshinone, hence named as tanshinol derivative. Protonated molecule $[\mathrm{M}+\mathrm{H}]^{+}$resolved at $24.1 \mathrm{~min}$ and at $\mathrm{m} / z 302$ yielded the product ions at $m / z 284\left[\mathrm{M}-\mathrm{H}_{2} \mathrm{O}\right], 256\left[\mathrm{M}-\mathrm{C}_{2} \mathrm{H}_{5} \mathrm{OH}\right], 161,123$ and 102, indicating it as an ester derivative, however exact structure could not be established.

Precursor ion $[\mathrm{M}+\mathrm{H}]^{+}$at $m / z 359$ yielded product ions at $m / z 341\left[\mathrm{M}-\mathrm{H}_{2} \mathrm{O}\right]^{+}, 327,309\left[-\mathrm{H}_{2} \mathrm{O}\right]^{+}, 295[-$ $\left.\mathrm{CH}_{2}\right]^{+}, 281[\mathrm{~m} / z 309-\mathrm{CO}]^{+}, 263\left[-\mathrm{H}_{2} \mathrm{O}\right]^{+}, 247,235[\mathrm{~m} / z 263-\mathrm{CO}], 215,189,171\left[\mathrm{~m} / z 215-\mathrm{CO}_{2}\right], 143[-\mathrm{CO}]$, 137,105 showed the presence of six oxygens and furan ring, hence assigned as tinosporin $\mathrm{B}$ comparing with reference spectra. Precursor ion $[\mathrm{M}+\mathrm{H}]^{+}$at $\mathrm{m} / z 413$ yielded product ion at $\mathrm{m} / z 251$ due to loss of glucose moiety of $162 \mathrm{Da}$. Further it generated molecular ions at $m / z 233\left[-\mathrm{H}_{2} \mathrm{O}\right]^{+}, 215\left[-\mathrm{H}_{2} \mathrm{O}\right]^{+}, 175$ [Aromatic], 159, 147 [-CO], 135, 121 and 109, the characteristic ions of tinocordifolioside. The molecular formula of molecular ion peak at $m / z 599.4156$ was calculated as $\mathrm{C}_{28} \mathrm{H}_{38} \mathrm{O}_{12} \mathrm{~S}$ that further generated product ions at $\mathrm{m} / z 581,535,469,385$, $361,298,247,193,167$ and 102 . The product ion at $m / z 361$ was formed due to loss of $\mathrm{C}_{8} \mathrm{H}_{14} \mathrm{O}_{6} \mathrm{~S}$. Comparing the fragmentation, it was tentatively assigned as cordifolide $\mathrm{A}$. The precursor ion $[\mathrm{M}+\mathrm{H}]^{+}$at $\mathrm{m} / z 251$ generated distinctive product ion at $m / z 233\left[\mathrm{M}-\mathrm{H}_{2} \mathrm{O}\right]^{+},\left[\mathrm{M}-2 \mathrm{H}_{2} \mathrm{O}-\mathrm{CO}\right]^{+} 175[\mathrm{~m} / z 203-\mathrm{CO}]^{+}, 159\left[-\mathrm{CH}_{4}\right]^{+}, 145,133$ $[-\mathrm{HCCH}]^{+}, 117\left[-\mathrm{CH}_{4}\right]^{+}$, which were the characteristic ions of the aromatic lactone, hence identified as tinocordifolin. Molecular formula for the parent ion $[\mathrm{M}+\mathrm{H}]^{+}$at $m / z 521$ was calculated as $\mathrm{C}_{26} \mathrm{H}_{33} \mathrm{O}_{11}$ with a difference of $13.93 \mathrm{ppm}$. Initial neutral loss of $162 \mathrm{Da}$ generated the minor ion peak at $\mathrm{m} / z 359$ that generated ion at $\mathrm{m} / z 313$ due to loss of $\mathrm{HCO}_{2}$. Further fragments at $\mathrm{m} / z$ 205, 163, 131 and 121 indicates its lactone nature and columbin, hence structure was established as columbin glycoside; also known as palmatoside C. Precursor ion $[\mathrm{M}+\mathrm{H}]^{+}$at $m / z 391$ yielded the product ions at $m / z 373\left[\mathrm{M}-\mathrm{H}_{2} \mathrm{O}\right]^{+}$and at $m / z 355,341,327,313$ due to loss of $\mathrm{H}_{2} \mathrm{O}, \mathrm{CH}_{3} \mathrm{OH}$, $\mathrm{CO}$ and $\mathrm{CH}_{3} \mathrm{COOH}, 259,121$ and 107. It was tentatively identified as salvinorin $\mathrm{B}$ when compared with reference standard.

Low-energy CID-MS/MS of the protonated molecule $[\mathrm{M}+\mathrm{H}]^{+}$at $m / z 307$ afforded the following products ion at $m / z 289,177,169,153,139$ and 121 and identified as (-)-gallocatechin when compared with reference standard. Precursor ion $[\mathrm{M}+\mathrm{H}]^{+}$at $m / z 315$ yielded product ions at $m / z 299\left[\mathrm{M}-\mathrm{CH}_{4}\right]^{+}, 269\left[\mathrm{M}-\mathrm{H}_{2} \mathrm{C}=\mathrm{O}\right]^{+}, 175,145$, 123,107 were in accordance to methoxy-5, $3^{\prime}, 4^{\prime}$-trihydroxyflavone. Precursor ion $[\mathrm{M}+\mathrm{H}]^{+}$at $m / z 373$ resolved at 28.4 min afforded product ions at $m / z 357\left[\mathrm{M}-\mathrm{CH}_{4}\right]^{+}, 341\left[\mathrm{M}-\mathrm{CH}_{4}-\mathrm{CH}_{3} \mathrm{OH}\right]^{+}, 329,317,287,205,167,137$ was identified as pentamethoxyflavone. Protonated molecule $[\mathrm{M}+\mathrm{H}]^{+}$resolved at $23.9 \mathrm{~min}$ and at $\mathrm{m} / z 1031$ yielded product ions at $m / z 869$ [M-Glu], 851, 743, 725 [M -Glu -Rhn], 705, 581 [M -Glu -Rhn -Rhn], 527, 415 [M -Glu -Rhn -Rhn -Glu]. Other product ions at $m / z$ 397, 301, 273, 253 were comparable with diosgenin, hence identified as diosgenin 3-[glucosyl-(1-> 4)-rhamnosyl-(1->4)-[rhamnosyl-(1->2)]-glucoside. Parent ion $[\mathrm{M}+\mathrm{H}]^{+}$at $m / z$ 415 resolved at 35.7 min yielded typical product ion at 135, 119, 109 similar to those lycopene, exact identification could not be done, hence named as lycopene derivative.

Precursor ion $[\mathrm{M}+\mathrm{H}]^{+}$at $m / z 230$ was identified as amino-tridecanoic acid as it yielded ions at $m / z 212$ [M $\left.-\mathrm{H}_{2} \mathrm{O}\right]^{+}, 195\left[\mathrm{M}-\mathrm{H}_{2} \mathrm{O}-\mathrm{NH}_{3}\right]^{+}, 184[\mathrm{~m} / z 212-\mathrm{CO}]^{+}, 167,155,141,127$ and 113 comparable to reference spectra. The pronated peak $[\mathrm{M}+\mathrm{H}]^{+}$at $\mathrm{m} / z 313$ was identified as 3-oxo-nonadecanoic acid, as it yielded product ions at $\mathrm{m} / \mathrm{z} 295,277,267,253,225,211,169,141,127$ and 113 in same fashion as amino-tridecanoic acid. Precursor ion $[\mathrm{M}+\mathrm{H}]^{+}$resolved at $27.2 \mathrm{~min}$ of $\mathrm{m} / z 555$ yielded products ions at $m / z 359,327,309,299,281,265,247,229,215$, $181,177,159,139$, and 124 . Product ions at $\mathrm{m} / z 327$ to 153 are the exact match with tetrahydroxy-octadecenoic acid; further initial loss of $196 \mathrm{Da}$ indicated the loss of $2 \mathrm{H}_{3} \mathrm{PO}_{4}$ molecules. Hence, it was identified as phospholipid. Precursor ion $[\mathrm{M}+\mathrm{H}]^{+}$at $m / z 387$ could not be identified. However, the product ions at $m / z 371,353$, $311,290,268,203,177,175,161,147,149,135,121$ confirmed its steroidal nature. Another pronated molecule $[\mathrm{M}+\mathrm{H}]^{+}$at $m / z 397$ was identified as stigmastan-3,5-diene by comparing with reference standard as it yielded product ions at $m / z 381,301,287,285,247,229,189,175,147,135,125,109$. Precursor ion $[\mathrm{M}+\mathrm{H}]^{+}$at $\mathrm{m} / z 495$ resolved at 26.5 yielded products ions at $m / z 441,423,371,357,339,357,339,329,311,301,283,233,219,179$, 145,113 . It was tentatively assigned as auricularine like compound by matching the fragmentation pattern with standard spectra. Phosphatidylcholine, choline, robinobiose, and glycosminine were identified using reference spectrum of these compounds.

Quantitative analysis of bioactive compounds. The developed method was used to quantify 5 major metabolites in 21 samples of $T$. cordifolia grown on different host trees. The selective ion monitoring mode in Q-TOFMS was used to monitor selective and specific ions fragmentation pattern of 5 compounds. Quantitative Mass Hunter (B.04.00) was used to develop calibration curves of standards at different concentrations. The correlation coefficient was determined by using a linear regression model. The limits of detection (LOD) and limits of quantification (LOQ) were measured with a signal-to noise ratio $(\mathrm{S} / \mathrm{N})$ of about 3 and 10 . The correlation coefficients $\mathrm{r}^{2}$ (0.9784-1.000), LOD (0.18-4.25 ng), and LOQ (6.03-15.63 ng) were observed for five standards used in the study (Table 4). Calibration curve for the standard compounds exhibited good linearity in the measured range of $0.25-50 \mathrm{ng} / \mathrm{mL}$. The coefficients of linear regression were determined from 5 independent experiments. The calibration curves by means of weighted $(1 / \mathrm{x} 2)$ least squares linear regression were $\mathrm{y}=0.24 \mathrm{x}+0.026$ and $r^{2}=0.9892$ for berberine, $y=0.029 x-0.0025$ and $r^{2}=0.9987$ for jatrorrhizine, $y=0.084 x+0.027$ and $r^{2}=0.9992$ for palmatine, $y=0.122 x+0.028$ and $r^{2}>1.000$ for choline and $y=0.0962 x+0.343$ and $r^{2}=0.9984$ for magnoflorine. On the basis of calibration curves, the contents of berberine, choline, palmatine, magnoflorine, and jatrorrhizine in T. cordifolia extracts were quantified (Table 5). AIN group was found to have highest content of jatrorrhizine $(2.88 \mathrm{ng} / \mathrm{mL})$ as noticed in the qualitative analysis. FBG group was found to be endowed with 


\begin{tabular}{|l|l|l|l|l|l|l|l|l|}
\hline S. No. & Compounds & $\begin{array}{l}\text { Test range } \\
(\mathbf{n g} / \mathbf{m l})\end{array}$ & $\begin{array}{l}\text { Linear range } \\
(\mathbf{n g} / \mathbf{m l})\end{array}$ & $\begin{array}{l}\mathbf{R t} \\
(\mathbf{m i n})\end{array}$ & $\mathbf{R}^{2}$ & Slope & $\begin{array}{l}\text { LOD } \\
(\mathbf{n g})\end{array}$ & $\begin{array}{l}\text { LOQ } \\
(\mathbf{n g})\end{array}$ \\
\hline 1. & Berberine & $1.2-1200$ & $1.9-200.6$ & 30.09 & 0.9892 & 7.9482 & 1.98 & 5.971 \\
\hline 2. & Palmatine & $1.2-1200$ & $2.9-201$ & 32.30 & 0.9992 & 7.9454 & 1.60 & 4.994 \\
\hline 3. & Jatrorrhizine & $0.4-1200$ & $2.1-199$ & 28.77 & 0.9987 & 8.0049 & 4.25 & 12.87 \\
\hline 4. & Magnoflorine & $0.6-1200$ & $2.7-201$ & 28.35 & 0.9984 & 7.9272 & 2.24 & 6.80 \\
\hline 5. & Choline & $2.0-1200$ & $4.6-200.8$ & 7.04 & 1.0000 & 7.9847 & 0.18 & 0.5289 \\
\hline
\end{tabular}

Table 4. Test range, linearity, retention time, regression, slope, LOD, and LOQ for the reference compounds.

\begin{tabular}{|l|l|l|l|l|l|l|l|l|}
\hline S. No. & Compounds & AIN & ALC & ALL & ANI & FBG & TMI & Control \\
\hline 1. & Berberine & 0.06 & 0.02 & 0.02 & 0.21 & 0.01 & 0.01 & 0.01 \\
\hline 2. & Palmatine & 1.34 & 4.16 & 1.81 & 1.78 & 2.21 & 1.97 & 2.23 \\
\hline 3. & Jatrorrhizine & 2.88 & 0.11 & 0.04 & ND & 0.10 & 0.13 & 1.31 \\
\hline 4. & Magnoflorine & 5.93 & 7.65 & 1.53 & 0.76 & 6.73 & 5.76 & 7.76 \\
\hline 5. & Choline & 4.45 & 5.12 & ND & 1.12 & 6.10 & 6.02 & 6.47 \\
\hline
\end{tabular}

Table 5. The concentrations of berberine, palmatine, jatrorrhizine, magnoflorine, and choline (ng/mL) in $T$. cordifolia climbed on different trees.

significantly high concentrations of magnoflorine and choline confirmed the validity of qualitative analysis. Choline, a major component of cell wall was found to be highest in control (T. cordifolia climbed on the steel pole). ALC was found to have highest levels of palmatine $(4.16 \mathrm{ng} / \mathrm{mL})$ (Table 5). Overall, quantitative analysis confirmed the authenticity of results obtained in qualitative analysis.

\section{Discussion}

To explore the chemoprofiles of T. cordifolia climbed on different trees, a simple but efficient HPLC-QTOF-MS based method was used. HPLC-QTOF-MS method is very precise having $>98 \%$ interday and intra-day accuracy. It has also recovered $>98 \%$ of seck its efficiency (Table 1 ). Both factors are important to standardize in order to minimize the variability and error rate across the data, in particular for qualitative analysis. Retention time alignment and mass data intensity normalization also affect the quality of analysis. Hence, the minimum difference in retention time and mass error noticed in this study were $<0.05 \mathrm{~min}$ and $<5 \mathrm{ppm}$ respectively that further enhanced the data quality. Multivariate analysis methods are strong tools to differentiate the variations across the data $^{21}$. In this study SVM and NB classifiers have been found to be the most accurate with $\mathrm{r}^{2}<0.97$. To decrease the false discovery rate, SNK post-hoc test with permutative $(n=50)$ Bonferroni FDR multiple testing correction was employed to increase the confidence in the analysis. The Venn diagram analysis has predicted the unique or the most abundant compounds across the different groups that can further be used as markers to identify the specific groups (Table 2).

T. cordifolia co-occurred with $A$. indica (AIN) contains 8-hydroxytinosporide and one unknown triterpenoid that can be used to differentiate this group from others. 5-Allyloxysalvigenin, trans-farnesol, reticuline and $\mathrm{N}$-isovaleroylglycine are found in the control ( $T$. cordifolia co-occuredwith steel pole), whereas, palmatoside C, D-glucan and 5-aminovaleric acid were found only in the ANI ( $T$. cordifolia co-occurred with A. nilotica). Other group specific compounds include tinocordifolioside, tinoridine, tinosporinone, baenzigeroside $\mathrm{A}$, tinosinen and 11-hydroxymustakone. All these compounds are found to be the group specific. However, medicinal importance of these compounds needs to be explored. Further fold change analysis have shown the highest variables in the T. cordifolia that co-occured with $A$. indica and steel pole or AIN and CON groups. Least number of variables are observed in TMI, ALL and FBG groups, i.e. T. cordifolia co-occured with T. indica, A. lebbeck and F. benghalensis respectively (Table 3 ). These qualitative results are further verified by quantitative analysis with some standard compounds. It is noticed that AIN contains the highest quantity of jatrorrhizine $(2.88 \mathrm{ng} / \mathrm{mL})$ and ALC group contains palmatine $(4.16 \mathrm{ng} / \mathrm{mL})$ and magnoflorine $(7.65 \mathrm{ng} / \mathrm{mL})$ (Table 5). The results of quantitative analysis are in accordance with qualitative analysis and have increased the confidence in the data. AIN group is also found to be a rich source of specific and up-regulated terpenoids including 8-hydroxytinosporide, unidentified terpenoids of $457 \mathrm{Da}$, borapetoside D, N-methylcoclaurine, isotanshinone II, oblongine, tinosporaside and cycloeucalenol, alkaloid jatrorrhizine and flavanoids i.e. peonidin, 5-allyloxysalvigenin and chrysin (Table 4). More specifically, AIN have increased contents of terpenoids along with specific alkaloid that synthesized from different route and fllvanoids.

Plants are chemically divergent and differe in biochemicals with plant species ${ }^{22-26}$. The plant chemistry mediates ecological interactions and influence the selection traits including specific constitutes ${ }^{27}$. Inter and intra-specific ecological interactions or competition within plant communities has been reported to influence the selection of an allelopathic secondary plant compound ${ }^{28}$. Hence, various kinds of selection forces may lead to different chemotypes within a plant species. In the current study, AIN group which is reported with the highest immunomodulatory activity is found to have high levels of terpenoids, jatrorhizine, flavonoids, coumarins, phytosterols and other categories compounds. The 8-hydroxytinosporide, scalar-17(25)-en-19-ol, borapetosides D, 
cycloartane-24,25-diol-3-one, palmarin, tinosporaside, and isotanshinone IIA are the terpenoids. One study has reported a significant decrease in concentrations of tinosporaside and berberine during winter season ${ }^{29}$. However, in the group AIN tinosporoside is found to be elevated as compared to other groups. High levels of borapetoside $\mathrm{D}$, isotanshinone II, cycloeucalenol, chrysin, and methylcoumarin are found in AIN. These metabolites have been reported to have antioxidant properties and increase glucose uptake $\mathrm{e}^{30-34}$. Tanshinone II, an analogue of isotanshinone II has been reported to have anti-fatigue properties by increasing the muscle glucose uptake and decreasing the lactic acid production ${ }^{35,36}$. Tanshinone II A is also found to be effective in treatment of cardiac problems despite of its less absorption through intestine ${ }^{37}$.

Concentrations of isoquinoline alkaloids i.e. N-methylcoclaurine, isoquinolone alkaloid, jatrorrhizine, oblongine, and haplopine are also found to be the highest in AIN group. N-methylcoclaurine inhibits the solute carrier organic anion transporter family member $1 \mathrm{~B} 1$ and $1 \mathrm{~B} 3^{38}$. It has also been established that jatrorrhizine, oblongine, $\mathrm{N}$-methylcoclaurine and magnoflorine are agonist of dopamine receptors ${ }^{39,40}$. Compounds that are monoamine depletors, could be useful to treat Parkinson or Huntington's disease. Interaction of these alkaloids with dopamine receptors also modulate c-AMP metabolic process and cytosolic calcium ion concentration that are important in regulation of energy balance and other metabolic processes ${ }^{41-43}$. These alkaloids being dopamine receptors agonists activate trigeminal motoneurons that increase the muscle tone ${ }^{44}$. These compounds along-with above reported terpenoids, flavonoids and coumarin are also capable to modulate MAPK, ERK1, ERK2 and protein kinase A that are involved in signal transduction pathways to control the innate and adaptive immunity and cell death ${ }^{45-47}$. Cinnamaldehyde and sodium thiosalicylate along-with 20-hydroxyecdysone elevated in AIN group are known anti-inflammatory compounds and boost up immune system ${ }^{4-50}$. Hence, synergistic effect of these compounds along with jatrorrhizine might be a possible reason of the highest immunodulatory activities of the crude extract of T. cordifolia co-occured with $A$. indica. However, the role of other compounds present in minute quantities cannot be ignored and need to studied carefully.

The present study has clearly established that different chemotypes of T. cordifolia are due to biotic interactions with other plants. Levels of choline contents is affected as observed in the present study by interspecific interactions and therefore, the cell membrane composition for the ease of working of interactive compounds from both species. Hence, T. cordifolia co-occured with A. indica have the best medicinal efficacy due to the presence of high concentrations of specific terpenoids, alkaloids, and flavonoids which affect ERK1, ERK2 and MAPK cascade, c-AMP metabolic process, cytosolic calcium ion concentration and angiogenesis. However, more studies are required to explore the individual or syngesitic mechanism of these compounds. The study also emphasizes the necessity to study secondary metabolites accumulation in the plants under normal and biotic interaction conditions. Identification and understanding of the biotic and abiotic factors which influence the secondary metabolites production in T. cordifolia may also help to increase its medicinal efficacy. Better understanding of the ecological interactions due to co-occurrence of T. cordifolia with other plants will help to increase its medicinal efficacy and also to understand physiology of plant.

\section{Methods}

Standard compounds and chemicals. Standard compounds, berberine, jatrorrhizine, palmatine, magnoflorine, choline, ecdysteroids, lidocaine, D-camphor and 5,7-isoflavone were purchased from the Sigma-Aldrich, St. Louis, MO, USA. Acetonitrile, formic acid and water of LC-MS grade were purchased from the Sigma-Aldrich.

T. cordifolia samples. Female T. cordifolia samples (protected from the use of any type of pesticides and organic material) were collected from a botanical garden, NRIBAS, Pune (with GPS location coordinates Latitude: $18.495^{\circ} \mathrm{N}$, Longitute: $73.8053^{\circ} \mathrm{E}$ ), in September 2011 . The three stem samples from four-year-old $T$. cordifolia co-occured with steel pole (Control), Azadirachta indica (Meliaceae) [AIN], Albizia lebbeck (Fabaceae) [ALL], Tamarindus indica (Fabaceae) [TMI], Ficus benghalensis (Moraceae) [FBG], Acacia nilotica (Fabaceae) [ANI], and Acacia leucophloea (Fabaceae) [ALC] were collected. The samples were authenticated and specimen samples were deposited in the herbarium division of NRIBAS, Pune (Voucher No; 1995).

Preparation of extracts. The stem samples were washed with tap water followed by deionized water to remove soil and other traces. These were dried in the air for 4 weeks. The air-dried stems were chopped and further converted into fine small pieces by mixer grinder (Philips, India). Dried stems ( $10 \mathrm{~g})$ was extracted overnight with deionized water (Direct-Q, Millipore) $(1: 1 \mathrm{w} / \mathrm{v})$ in orbital shaker at $37^{\circ} \mathrm{C}$ and $180 \mathrm{rpm}$ to yield the thick juice. Extracts were then centrifuged at $15,000 \mathrm{~g}$ for $10 \mathrm{~min}$ at $4^{\circ} \mathrm{C}$. The extraction was repeated three times for each sample and the supernatant was collected. The percolate from three repeats of each sample was then concentrated in a rotary vacuum evaporator at $50-60^{\circ} \mathrm{C}$. The supernatant juice was quick-freezed at $-80^{\circ} \mathrm{C}$ (Thermo-Fisher, Germany) and lyophilized (Freezone 4.5 Labconco, CA, USA) to yield a dry homogenous powder $(0.3 \mathrm{~g})$ and stored at $-80^{\circ} \mathrm{C}$. The lyophilized powder from various samples was reconstituted in LC-MS grade water to make a solution of $5.0 \mathrm{mg} / \mathrm{ml}$. Solutions were vortexed and centrifuged at $15,000 \mathrm{~g}$ for 20 minutes at $4^{\circ} \mathrm{C}$ temperature. The supernatants were carefully removed and filtered through $0.22 \mu \mathrm{m}$ syringe filters and transferred to 96 well plates. The complete workflow of the study design is depected in Fig. S1.

HPLC-ESI-QTOF-MS. T. cordifolia stem extracts were resolved over ZORBAX Eclipse Plus reversed phase column $(\mathrm{C} 18,2.1 \times 250 \mathrm{~mm})$ having particle size $5 \mu \mathrm{m}$. Auto-sampler and column temperatures were maintained at $4{ }^{\circ} \mathrm{C}$ and $40^{\circ} \mathrm{C}$. Injection volume was kept constant, i.e. $20 \mu \mathrm{l}$ for all samples. Chromatographic separation was carried out with Agilent 1200 Series HPLC interfaced to an Agilent 6520 Accurate-Mass QTOF-MS, with mobile phase A (water containing $0.1 \%$ formic acid) and B (acetonitrile containing $0.1 \%$ formic acid). The gradient program was carried as follows: 0-8 min, $5 \% \mathrm{~B}$; 9-15 min, 5-20\% B; $16-22 \mathrm{~min}, 20-45 \% \mathrm{~B} ; 23-30 \mathrm{~min}, 45-65 \%$ B; 31-35 min, 65-90\% B; 36-40 min, 90-5\% B; and 41-45 min, 5\% B. Flow rate of $0.5 \mathrm{ml} / \mathrm{min}$ was kept constant. 
Q-TOF-MS was operated in a positive ion polarity mode and extended dynamic range $(1700 \mathrm{~m} / \mathrm{z}, 2 \mathrm{GHz})$ with following parameters: gas temperature $350^{\circ} \mathrm{C}$, nebulizer $50 \mathrm{Psi}$, gas flow $11 \mathrm{~L} / \mathrm{Min}$, capillary voltage $3500 \mathrm{~V}$, fragmentor voltage $175 \mathrm{~V}$, nozzle $500 \mathrm{~V}$, skimmer voltage $65 \mathrm{~V}$, octapole RF $250 \mathrm{~V}$ and octapole DC1 $48 \mathrm{~V}$. Accurate MS/MS spectra were acquired in the range $100-1100 \mathrm{~m} / z$ with acquisition rate 3 spectra $\mathrm{s}^{-1}$. To assure the mass accuracy of recorded data, standards of lidocaine $(234.3 \mathrm{~m} / \mathrm{z})$ and 5,7 -isoflavone $(284.3 \mathrm{~m} / \mathrm{z})$ were infused with samples along with continuous internal calibration. Overlay TIC of all the extracts depicting changes in the metabolites between various groups is represented in Fig. S2.

Quality assurance. Standard compound mixture of $5 \mathrm{ppm}$ was injected to check the operational conditions of the MS before sample analysis. Analogue compounds of standards in samples were identified on the basis of their retention time and the product ions. Quality control of extracts was also performed by mixing the three extracts from individual groups so that actual levels of metabolites can be compared with the individual sample of that group. Another quality control check was kept from mixing the extracts with standards (2.5 ppm) to check the peak volumes and intensity. After identification, comparative concentration of the analytes was calculated by its area and intensity ratio.

Multivariate statistical analysis. MassHunter (Qualitative Analysis Version B.04.00) and Profinder (Version B.06.00) softwares (Agilent Technologies) were used to process raw mass data. Molecular features presents in raw data sets were extracted keeping minimum ion abundance 5000 cycles per sec (cps), peak space tolerance $0.0025 \mathrm{~m} / z$ or $5 \mathrm{ppm}$ and relative height $2.5 \%$ (Fig. S3). Processed data sets were imported into the Mass Profiler Professional (MPP) software (Version.B.02.02; Agilent Technologies) and aligned using retention time window and mass difference $0.2 \mathrm{~min}$ and $5.0 \mathrm{ppm}$. A molecular feature present in less than 3 samples of a group and having $\mathrm{p}>0.05$, fold change $\leq 2.0$ and $\mathrm{CV}>15$ were removed from datasets. Refined data were further subjected to multivariate analysis to prepare five different discrimination models, namely partial least square discrimination analysis (PLS-DA), Support Vector Machines (SVMs), Naive Bayes (NB), Decision Tree (DT), and Neural network (NN). All the models were trained and used for further identification of unknown samples. Discriminated data sets from best model were subjected to statistical analysis using One-way ANOVA Student-Newman-Keuls (SNK) post-hoc test with Bonferroni false discovery rate (FDR) multiple testing correction and asymptotic $\mathrm{p}$ values were computed. Principal component analysis (PCA) was performed using the molecular feature (metabolites) having $\mathrm{p}<0.001$ and fold change $>2.0$ in at least one group. Finally, all the metabolites having any missing value were removed.

Quantitation of selected compounds using Selected lon Monitoring (SIM). All the standards and extracts were subjected to selected ion monitoring with defined collision energies. For the quantification the transitions at $\mathrm{m} / z 342.16 \rightarrow 297.03$ for magnoflorine, $\mathrm{m} / z 336 \rightarrow 320$ for berberine, at $\mathrm{m} / z 352 \rightarrow 336$ for palmatine, at $m / z 338 \rightarrow 322$ for jatrorrhizine, and at $m / z 146.13 \rightarrow 86.93$ for choline were monitored in SIM mode of Q-TOF-MS keeping scan time $0.3 \mathrm{~s}$ per transition. Varying concentrations of standards were used to construct standard curves and metabolites in the extracts were quantified by using Agilent MassHunter Quantitative Analysis Software (Version B.02.00). A window of 100 ppm was set for fragment identification. Standards and their corresponding metabolites in the extracts were quantified using the peak size of the fragment by extracting ion chromatogram function.

\section{References}

1. Panchabhai, T. S., Kulkarni, U. P. \& Rege, N. N. Validation of therapeutic claims of Tinospora cordifolia: A review. Phytotherapy Research 22, 425-441 (2008).

2. Bishayi, B., Roychowdhury, S., Ghosh, S. \& Sengupta, M. Hepatoprotective and immunomodulatory properties of Tinospora cordifolia in CCl4 intoxicated mature albino rats. J. Toxicol. Sci. 27, 139-146 (2002).

3. Rawal, A. K., Muddeshwar, M. G. \& Biswas, S. K. Rubia cordifolia, Fagonia cretica linn and Tinospora cordifolia exert neuroprotection by modulating the antioxidant system in rat hippocampal slices subjected to oxygen glucose deprivation. BMC Complement. Altern. Med. 4 (2004).

4. Gupta, R. \& Sharma, V. Ameliorative effects of Tinospora cordifolia root extract on histopathological and biochemical changes induced by aflatoxin-b(1) in mice kidney. Toxicol. Int. 18, 94-8 (2011).

5. Mittal, A. \& Dabur, R. Detection of new human metabolic urinary markers in chronic alcoholism and their reversal by aqueous extract of Tinospora cordifolia stem. Alcohol Alcohol. 50, 271-281 (2015).

6. Shirolkar, A., Sharma, B., Lata, S. \& Dabur, R. Guduchi Sawras (Tinospora cordifolia): An Ayurvedic drug treatment modulates the impaired lipid metabolism in alcoholics through dopaminergic neurotransmission and anti-oxidant defense system. Biomed. Pharmacother. 83, 1265-1277 (2016).

7. Thatte, U. M. \& Dahanukar, S. A. Comparative study of immunomodulating activity of Indian medicinal plants, lithium carbonate and glucan. Methods Find. Exp. Clin. Pharmacol (1988).

8. Narkhede, A. N., Jagtap, S. D., Kasote, D. M., Kulkarni, O. P. \& Harsulkar, A. M. Comparative immunomodulation potential of Tinospora cordifolia (Willd.) Miers ex Hook. F., Tinospora sinensis (Lour.) merrill and Tinospora cordifolia growing on Azadirachta indica A. Juss. Indian J. Exp. Biol. 52, 808-813 (2014).

9. Gahlaut, A., Gothwal, A. \& Dabur, R. TLC Based Analysis of Allelopathic Effects on Tinosporoside Contents in Tinospora cordifolia. J. Chem. Pharm. Res. 4, 3082-3088 (2012).

10. Chen, X. F., Wu, H. T., Tan, G. G., Zhu, Z. Y. \& Chai, Y. F. Liquid chromatography coupled with time-of-flight and ion trap mass spectrometry for qualitative analysis of herbal medicines. J. Pharm. Anal. 1, 235-245 (2011).

11. Roessner, U. Metabolic Profiling Allows Comprehensive Phenotyping of Genetically or Environmentally Modified Plant Systems. Plant Cell Online 13, 11-29 (2001)

12. Shirolkar, A., Gahlaut, A., Hooda, V. \& Dabur, R. Phytochemical composition changes in untreated stem juice of Tinospora cordifolia (W) Mier during refrigerated storage. J. Pharm. Res. 7, 1-6 (2013).

13. Misaki, M., Kim, Y., Bandettini, P. A. \& Kriegeskorte, N. Comparison of multivariate classifiers and response normalizations for pattern-information fMRI. Neuroimage 53, 103-118 (2010).

14. Byvatov, E. \& Schneider, G. Support vector machine applications in bioinformatics. Appl. Bioinformatics 2, 67-77 (2003).

15. Massbank, http://www.massbank.jp/SearchPage.html Accessed on 30-12-2017. 
16. Metalin, https://metlin.scripps.edu/landing_page.php?pgcontent=simple_search Accessed on 30-12-2017.

17. Inada, A., Murayta, H. \& Inatomi, Y. T. N. Cycloartane Triterpenes From the Leaves of Aglaia Harmsiana. J. Nat. Prod. 58, 1143-1146 (1995).

18. Hanuman, J. B., Bhatt, R. K. \& Sabata, B. A clerodane furano-diterpene from Tinospora cordifolia. J. Nat. Prod. 51, 197-201 (1988).

19. Fukuda, N., Yonemitsu, M. \& Kimura, T. Studies on the constituents of the stems of Tinospora tuberculata, V. Isolation and structure elucidation of the new furanoid diterpene glucoside borapetoside H. Liebigs Ann. 1995, 1689-1691 (1995).

20. Shinozaki, J., Shibuya, M., Ebizuka, Y. \& Masuda, K. Cyclization of All- E - and 2 Z -Geranylfarnesols by a Bacterial Triterpene Synthase: Insight into Sesterterpene Biosynthesis in Aleuritopteris Ferns. Biosci. Biotechnol. Biochem. 77, 2278-2282 (2013).

21. Rajalahti, T. \& Kvalheim, O. M. Multivariate data analysis in pharmaceutics: A tutorial review. International Journal of Pharmaceutics 417, 280-290 (2011).

22. Kliebenstein, D. J. Genetic Control of Natural Variation in Arabidopsis Glucosinolate Accumulation. PLANT Physiol. 126, 811-825 (2001).

23. Macel, M., Vrieling, K. \& Klinkhamer, P. G. L. Variation in pyrrolizidine alkaloid patterns of Senecio jacobaea. Phytochemistry 65 , $865-873$ (2004).

24. Witte, L., Ernst, L., Adam, H. \& Hartmannt, T. Chemotypes of two pyrrolizidine alkaloid-containing Senecio species. Phytochemistry 31, 559-565 (1992).

25. Linhart, Y. B. \& Thompson, J. D. Thyme is of the essence: Biochemical polymorphism and multi-species deterrence. Evol. Ecol. Res. 1, 151-171 (1999).

26. Van Leur, H., Vet, L. E. M., Van Der Putten, W. H. \& Van Dam, N. M. Barbarea vulgaris glucosinolate phenotypes differentially affect performance and preference of two different species of lepidopteran herbivores. J. Chem. Ecol. 34, 121-131 (2008).

27. Strauss, S. Y. \& Irwin, R. E. Ecological and Evolutionary Consequences of Multispecies Plant-Animal Interactions. Annu. Rev. Ecol. Evol. Syst. 35, 435-466 (2004).

28. Lankau, R. A. \& Strauss, S. Y. Mutual feedbacks maintain both genetic and species diversity in a plant community. Science. 317, 1561-1563 (2007).

29. Bajpai, V. et al. Chemometric Based Identification and Validation of Specific Chemical Markers for Geographical, Seasonal and Gender Variations in Tinospora cordifolia Stem using HPLC-ESI-QTOF-MS. Analysis. Phytochem. Anal. 28, 277-288 (2017).

30. Nabavi, S. F. et al. Neuroprotective effects of chrysin: From chemistry to medicine. Neurochemistry International 90, 224-231 (2015).

31. Wang, S. et al. Chrysin inhibits foam cell formation through promoting cholesterol efflux from RAW264.7 macrophages. Pharm. Biol. 53, 1481-1487 (2015).

32. Gao, S. et al. Cardiovascular actions and therapeutic potential of tanshinone IIA. Atherosclerosis 220, 3-10 (2012).

33. Rauf, A. et al. Suppression of inflammatory response by chrysin, a flavone isolated from Potentilla evestita Th. Wolf. in silico predictive study on its mechanistic effect. Fitoterapia 103, 129-135 (2015).

34. Kumar, S. et al. Novel thiocoumarins as inhibitors of TNF- $\alpha$ induced ICAM-1 expression on human umbilical vein endothelial cells (HUVECs) and microsomal lipid peroxidation. Bioorganic Med. Chem. 13, 1605-1613 (2005).

35. Xu, S. \& Liu, P. Tanshinone II-A: new perspectives for old remedies. Expert Opin Ther Pat 23, 149-153 (2013).

36. Lin, C. Y. et al. Antifatigue properties of tanshinone IIA in mice subjected to the forced swimming test. Pharm. Biol. https://doi.org /10.1080/13880209.2017.1401648 (2017).

37. Hong, H. J., Liu, J. C., Cheng, T. H. \& Chan, P. Tanshinone IIA attenuates angiotensin II-induced apoptosis via Akt pathway in neonatal rat cardiomyocytes. Acta Pharmacol. Sin. 31, 1569-1575 (2010).

38. De Bruyn, T. et al. Structure-Based Identification of OATP1B1/3 Inhibitors. Mol. Pharmacol. 83, 1257-1267 (2013).

39. Kosaraju, J. et al. Neuroprotective effect of Tinospora cordifolia ethanol extract on 6-hydroxy dopamine induced Parkinsonism. Indian J. Pharmacol. 46, 176-180 (2014).

40. Li, C. \& Wang, M.-H. Potential Biological Activities of Magnoflorine: A Compound from Aristolochia debilis Sieb. et Zucc. Korean J. Plant Resour. 27, 223-228 (2014).

41. Yuan, J. Y. et al. The effects of jatrorrhizine on contractile responses of rat ileum. Eur. J. Pharmacol. 663, 74-79 (2011).

42. Kong, L. D., Cheng, C. H. K. \& Tan, Ren Xiang. Monoamine oxidase inhibitors from rhizoma of Coptis chinensis. Planta Med. 67, 74-76 (2001).

43. Patel, M. B. \& Mishra, S. Hypoglycemic activity of alkaloidal fraction of Tinospora cordifolia. Phytomedicine, https://doi. org/10.1016/j.phymed.2011.05.006 (2011).

44. Hemsley, K. M. \& Crocker, A. D. Changes in muscle tone are regulated by D1 and D2 dopamine receptors in the ventral striatum and D1 receptors in the substantia nigra. Neuropsychopharmacology 25, 514-526 (2001).

45. Zhang, L. et al. SN38 increases IL-8 expression through the MAPK pathways in HCT8 cells. Int. J. Mol. Med. 39, 217-222 (2017).

46. Nam, K. W., Je, K. H., Shin, Y. J., Sam, S. K. \& Mar, W. Inhibitory effects of furoquinoline alkaloids from Melicope confusa and Dictamnus albus against human phosphodiesterase 5 (hPDE5A) in vitro. Arch. Pharm. Res. 28, 675-679 (2005).

47. Jeong, G. S. et al. Neuroprotective effects of constituents of the root bark of Dictamnus dasycarpus in mouse hippocampal cells. Arch. Pharm. Res. 33, 1269-1275 (2010).

48. Subash Babu, P., Prabuseenivasan, S. \& Ignacimuthu, S. Cinnamaldehyde-A potential antidiabetic agent. Phytomedicine 14, 15-22 (2007).

49. Chao, L. K. et al. Cinnamaldehyde inhibits pro-inflammatory cytokines secretion from monocytes/macrophages through suppression of intracellular signaling. Food Chem. Toxicol. 46, 220-231 (2008).

50. Gorelick-Feldman, J. I. Phytoecdysteroids: understanding their anabolic activity. Doctoral dissertation, Rutgers University-Graduate School-New Brunswick (2009).

\section{Acknowledgements}

Authors are thankful to Department of Science and Technology, Haryana and Department of Science and Technology, India for the financial support to carry out the work wide grant no HSCST/926 and FIST program respectively.

\section{Author Contributions}

R.D. and B.S. have done the experimental work. A.Y. has helped in re-collection and re-analysis of MS profiles to revalidate the LOD and LOQ values. R.D. led the project, data interpretation and prepared the manuscript.

\section{Additional Information}

Supplementary information accompanies this paper at https://doi.org/10.1038/s41598-019-50801-0.

Competing Interests: The authors declare no competing interests.

Publisher's note Springer Nature remains neutral with regard to jurisdictional claims in published maps and institutional affiliations. 
(c) (i) Open Access This article is licensed under a Creative Commons Attribution 4.0 International License, which permits use, sharing, adaptation, distribution and reproduction in any medium or format, as long as you give appropriate credit to the original author(s) and the source, provide a link to the Creative Commons license, and indicate if changes were made. The images or other third party material in this article are included in the article's Creative Commons license, unless indicated otherwise in a credit line to the material. If material is not included in the article's Creative Commons license and your intended use is not permitted by statutory regulation or exceeds the permitted use, you will need to obtain permission directly from the copyright holder. To view a copy of this license, visit http://creativecommons.org/licenses/by/4.0/.

(c) The Author(s) 2019 